\title{
Acceptance versus reappraisal: Behavioral, autonomic, and neural effects
}

\author{
Philippe R. Goldin ${ }^{1}$. Craig A. Moodie ${ }^{2}$. James J. Gross ${ }^{2}$
}

Published online: 17 January 2019

(C) The Psychonomic Society, Inc. 2019

\begin{abstract}
Emotion regulation (ER) is an important skill for well-being. Cognitive reappraisal is a goal-oriented cognitive change strategy. Acceptance involves decentering from immediate habits of reactivity, observing moment-to-moment shifts in thoughts, emotions, and sensations. These two regulation strategies are thought to have different effects on emotion; however, no study has examined the differential effects of reappraisal and acceptance on behavioral, autonomic, and brain responses in the context of ideographic personally salient negative self-beliefs. Thirty-five right-handed, healthy adults were presented idiographic negative self-beliefs embedded in autobiographical scripts. We measured negative emotion ratings, autonomic psychophysiology, and functional magnetic resonance imaging blood oxygen-level dependent responses while participants read neutral statements, reacted to their own negative self-beliefs, and implemented reappraisal and acceptance strategies. Compared with react, reappraisal resulted in significantly lesser negative emotion and respiration rate; no differences in heart rate and skin conductance level; greater brain responses implicated in cognitive control, language, and social cognition; and lesser amygdala responses. Compared with react, acceptance resulted in significantly lesser negative emotion, respiration rate, and heart rate; no difference in skin conductance level; and greater brain responses in networks implicated in cognitive control and attention. Compared with acceptance, reappraisal resulted in significantly lesser negative emotion; no difference in respiration rate and skin conductance level; higher heart rate; greater brain responses in brain regions implicated in cognitive control; and lesser brain responses in amygdala. Reappraisal is more effective than acceptance in down-regulating negative emotion, but may require greater recruitment of autonomic, cognitive, and brain resources.
\end{abstract}

ClinicalTrials.gov identifier: NCT02036658

Keywords Acceptance $\cdot$ Emotion regulation $\cdot \mathrm{fMRI} \cdot$ Reappraisal $\cdot$ Mindful attention $\cdot$ Neuroimaging

The ability to regulate emotions is a key skill for maintaining both mental and physical health (Gross, 2002, 2015). There are many opportunities in everyday life to regulate both negative and positive emotions that arise in response to external stimuli and situations. However, modulating long-held patterns of emotional reactivity to internally generated personally salient negative self-beliefs is arguably one of the most important contexts for emotion regulation. Negative self-beliefs can powerfully shape our relationship to others and to ourselves. They are a fundamental target of all psychological interventions that address mental disturbances.

Philippe R. Goldin

philippegoldin@gmail.com

1 University of California, Davis, Sacramento, CA 95817, USA

2 Stanford University, Stanford, CA, USA
There are many ways to regulate emotions, and these strategies vary in how difficult they are to implement and how effective they are at achieving emotion regulation goals. Cognitive reappraisal and acceptance are two adaptive emotion regulation strategies that both involve cognitive and attention processes. Importantly, they are thought to vary in terms of their effectiveness, implementation effort, and impact on multiple indicators of emotion reactivity, including experiential, autonomic psychophysiological, and brain responses. A recent meta-analysis of emotion regulation (Aldao, Nolen-Hoeksema, \& Schweizer, 2010) found across 114 studies that adaptive strategies (i.e., acceptance, reappraisal, and problem solving) were less likely to be used than maladaptive emotion regulation strategies (i.e., avoidance, rumination, suppression). Furthermore, in an adult community sample, adaptive strategies (e.g., acceptance, reappraisal, problem solving) were implemented more frequently than were maladaptive strategies (e.g., avoidance, self-criticism, hiding expression, suppression 
of experience, worry, rumination) across eight different emotion-eliciting situations (Aldao \& Nolen-Hoeksema, 2012). These findings highlight the frequency of use and mental health significance of reappraisal and acceptance.

However, contextual models of emotion regulation (Aldao \& Nolen-Hoeksema, 2012) highlight the importance of investigating how context influences the implementation of emotion regulation. In particular, there is much research on emotion regulation of experimenter-selected stimuli. In contrast, however, use of person-specific autobiographical situations with personally salient stimuli (e.g., negative self-beliefs) may be more salient for understanding how context influences emotion regulation processes.

\section{Cognitive reappraisal}

Cognitive reappraisal is a type of emotion regulation strategy that involves volitional reframing of the meaning of an emotion-eliciting stimulus (Gross, 2013). Reappraisal is a complex and sometimes effortful cognitive change strategy that relies on the interaction of multiple component processes, including cognitive control, working memory, monitoring, attention regulation, perspective taking, and linguistic processing. Reappraisal has been shown to reduce negative emotional reactivity, reduce autonomic hyperactivity, modulate emotion regulatory brain networks, and enhance physical and mental well-being (Goldin, McRae, Ramel, \& Gross, 2008; Gross, 2002, 2015).

Neuroimaging studies of reappraisal of emotion in healthy adults (Buhle et al., 2013; Ochsner, Silvers, \& Buhle, 2012) show increased recruitment of brain networks implicated in cognitive control (bilateral dorsolateral and ventrolateral prefrontal cortex [DLPFC, VLPFC], dorsomedial prefrontal cortex [DMPFC] and supplemental motor area [SMA]), attention (precuneus, superior parietal lobule, right DLPFC, and dorsal anterior cingulate cortex; Fan, McCandliss, Fossella, Flombaum, \& Posner, 2005) and linguistic processing (left VLPFC, left posterior middle temporal gyrus (MTG), and left angular/supramarginal gyrus). These brain networks work together in an integrated fashion to support the implementation of reappraisal.

To identify these reappraisal-related brain networks, most studies have used experimenter-selected stimuli such as faces, words, and pictures to elicit emotional reactivity in research participants. More recently, there is growing interest in using more ecologically valid stimuli. One method is to use participant-selected (i.e., idiographic) negative self-beliefs embedded in highly personally relevant contexts to examine the effects of reappraisal on emotional responses (i.e., behavioral, autonomic, and brain). Using this approach in a prior fMRI study, we examined reappraisal used to down-regulate emotional reactivity to negative self-beliefs embedded in personally salient autobiographical situations in healthy adults. We found brain network activations implicated in reappraisal, linguistic and visual processing, as well as significant inverse functional connectivity characterized by greater activity in reappraisalrelated PFC regions coupled with lesser amygdala activity (Goldin, Manber-Ball, Werner, Heimberg, \& Gross, 2009). This study also examined the temporal dynamics of fMRI BOLD responses in multiple PFC regions. Findings suggested that early cognitive control activity in DMPFC, DLPFC, and dorsal anterior cingulate cortex may diminish over time after reappraisal has been implemented to down-regulate emotional reactivity. Thus, there is preliminary evidence for the effectiveness of reappraisal of ideographic negative self-beliefs in personally salient autobiographical contexts. However, no studies have investigated the neural bases of reappraisal versus other types of emotion regulation strategies in the context of idiographic negative self-beliefs.

\section{Acceptance}

In the context of this study, acceptance refers to a specific orientation or cognitive stance toward ongoing mental and sensory experience. Acceptance is defined as a decentered observer perspective characterized by an open, curious, nonelaborative, and nonevaluative perspective. Acceptance involves the capacity to discern thoughts, emotions and sensations as transient events, which is thought to diminish automatic habitual patterns of emotional reactivity. Similar to the perspective that views negative feelings as mental events that are psychologically distanced from the self (Ayduk \& Kross, 2008; Kross \& Ayduk, 2008), we characterize acceptance of present-moment experience as one specific component of the larger construct of "decentering" that may be considered a multifaceted cognitive process related to mindful attention.

In contrast to reappraisal, acceptance does not involve reinterpretation, disputation, reasoning, language, inhibitory control, and response inhibition. Furthermore, acceptance is not a passive resignation (Garnefski, Kraaij, \& Spinhoven, 2002; Hofmann, Heering, Sawyer, \& Asnaani, 2009), but rather an active willingness to experience thoughts, emotions, and sensations while engaging in goal-directed behavior (Hayes, Strosahl, \& Wilson, 1999). Acceptance differs from reappraisal in its focus on awareness of the patterns of emotion reactivity rather than actively reframing the meaning of an emotion-eliciting stimulus (Liverant, Brown, Barlow, \& Roemer, 2008; Wolgast, Lundh, \& Viborg, 2011). Thus, while acceptance and reappraisal may share some basic attentional processes, they are distinct emotion-regulation strategies.

Studies of acceptance have begun to elucidate the effects of this ER strategy on emotion processes. In one study of emotional pictures, acceptance produced greater positive emotions and emotional expressivity and lesser respiration rate compared 
with no active emotion regulation (Dan-Glauser \& Gross, 2015). Neuroimaging studies have begun to investigate the brain correlates of acceptance of emotional reactivity. One fMRI study of healthy adults found that emotion acceptance versus viewing sad images produced greater fMRI BOLD responses in dorsomedial, dorsolateral, and ventrolateral PFC, and dorsal anterior midcingulate (Smoski et al., 2015). An fMRI study in university students (Lebois et al., 2015) found that, compared with immersion with stressful events, mindful attention (i.e., decentering or disengaging the self from the event) yielded greater brain responses in brain areas associated with perspective shifting and effortful attention (lateral and medial PFC, angular gyrus, and inferior and middle occipital gyrus). A recent fMRI study of adult females diagnosed with generalized anxiety disorder found that in contrast to cued worrying, emotion acceptance toward personally relevant worry statements resulted in lower ratings of distress and produced increased dorsal anterior cingulate cortex (dACC) and DMPFC activation and increased ventrolateral prefrontal cortex (VLPFC)-amygdala functional connectivity (Ellard, Barlow, Whitfield-Gabrieli, Gabrieli, \& Deckersbach, 2017). Importantly, in contrast to acceptance, a contrasting ER strategy, suppression of experiencing emotion, resulted in equivalent distress ratings and amygdala activation, but significantly greater insula and VLPFC activation, and decreased VLPFCamygdala functional connectivity. This suggests that emotion acceptance is distinguished from emotion suppression with respect to visceral reactivity (insula) and cognitive modulation of emotional reactivity (VLPFC-amygdala functional connectivity) in the context of adults with generalized anxiety disorder.

Acceptance has also been compared with other types of emotion regulation strategies. A recent study of seven distinct emotion regulation strategies across eight different situations found that acceptance was the most frequently implemented emotion regulation strategy and was the adaptive emotion regulation strategy that most predicted lesser psychopathology (Aldao \& Nolen-Hoeksema, 2012). This aligns with a model developed by Hayes et al. (1999) that proposes acceptance as an important strategy for regulating emotions across many situations when implemented flexibly and in a contextsensitive manner.

\section{Comparison of cognitive reappraisal and acceptance}

Few studies have directly compared the effects of acceptance and reappraisal. In one study by Hofmann et al. (2009), acceptance and reappraisal produced equivalent decreases in physiological arousal as indexed by heart rate during an impromptu speech. However, reappraisal resulted in greater reduction of self-reported anxiety. In a study that examined reactivity to negative affect inducing film clips, acceptance and reappraisal produced similar decreases in subjective distress, behavioral avoidance, and physiological reactions (Wolgast et al., 2011). In a study of reactivity to sad images, compared with acceptance, reappraisal resulted in greater reduction of negative emotion (Smoski et al., 2015). A study of clinical and nonclinical participants concluded that acceptance and reappraisal are partly overlapping emotion regulation strategies that share some subprocesses (e.g., attention regulation) but consist of different psychological functions and properties (Wolgast, Lundh, \& Viborg, 2013).

Neurally, there is initial evidence of distinct neural recruitment for reappraisal and acceptance ER strategies. An fMRI study of healthy adults found that, compared with reappraisal of emotional reactivity to sad images, acceptance produced greater BOLD responses in left insular cortex and left precentral gyrus, and lesser BOLD responses in frontal pole, ventromedial, and ventrolateral PFC regions (Smoski et al., 2015).

\section{The present study}

Our goal was to examine the differential impact of two distinct ER strategies - cognitive reappraisal and acceptance-on multiple indicators of emotional reactivity, including selfreported negative emotion ratings, autonomic psychophysiology (respiration rate, heart rate, skin conductance), and fMRI BOLD responses in 35 healthy adults. To do this, we induced emotional reactivity with participant-generated personally salient negative self-beliefs embedded in anxiety-related autobiographical situations. We expected that, compared with reacting to negative self-beliefs, both reappraisal and acceptance would result in decreases in self-reported negative emotion, heart rate, respiration rate, skin conductance, and amygdala responses. We also expected greater fMRI BOLD responses in cognitive control-related prefrontal cortex brain responses in dACC, DMPFC, VLPFC, DLPFC during reappraisal, and greater attention regulation-related responses in $\mathrm{dACC}$, precuneus, and superior and inferior parietal lobule during acceptance. For the direct comparison of reappraisal versus acceptance, however, we expected greater decreases in self-reported negative emotion, autonomic psychophysiology and amygdala, but greater fMRI BOLD responses in cognitive control-related PFC and left-lateralized language processing brain regions for reappraisal versus acceptance.

\section{Methods and materials}

\section{Participants}

We phone-screened 134 potential participants, and then administered 63 diagnostic interviews. We identified 53 eligible healthy adults who had no history of meeting the criteria of the 
Diagnostic and Statistical Manual of Mental Disorders, Fourth Edition (DSM-IV; American Psychiatric Association, 1994) for Axis I psychiatric disorders. Eighteen individuals did not complete all assessments required for entry to the study and were not included in our final sample of 35 healthy individuals. As shown in Table 1, participants were young-tomiddle age (range: 22.6-55.4 years of age), 57\% female, college educated, primarily Caucasian and Asian, middle class, and predominantly not living with a partner $(66 \%)$. All participants provided informed consent in accordance with the Institutional Review Board at Stanford University. This study was preregistered at NIMH (ClinicalTrials.gov identifier: NCT02036658).

\section{Inclusion/exclusion criteria}

All participants passed an MRI safety screen, were 21-55 years of age, fluent in English, right-handed as assessed by the Edinburgh Handedness Inventory (Oldfield, 1971), had no history of pharmacotherapy or psychotherapy (except for couple's therapy), and had no history of medical disorders, head trauma, and neurological disorders. Participants were excluded if they reported previous participation in meditation retreats, mindfulness-based stress reduction (MBSR) or history of regular meditation practice of $10 \mathrm{~min}$ or more, three or more times per week.

Table 1 Characteristics of participants

\begin{tabular}{ll}
\hline Variable & HC \\
& $n=35$ \\
\hline Gender (males, $n, \%)$ & $15(42.9 \%)$ \\
Age (years, $M \pm S D)$ & $32.2 \pm 8.9$ \\
Education (years, $M \pm S D)$ & $17.6 \pm 2.8$ \\
Ethnicity $(n, \%)$ & \\
Caucasian & $17(48.6 \%)$ \\
Asian & $12(34.3 \%)$ \\
Latino/a & $3(8.6 \%)$ \\
Multiple & $3(8.6 \%)$ \\
Yearly income $(\$ 1,000, n, \%)$ & \\
$<10 \mathrm{k}$ & $1(2.9 \%)$ \\
$10-25 \mathrm{k}$ & $3(8.6 \%)$ \\
$25-50 \mathrm{k}$ & $8(22.9 \%)$ \\
$50-75 \mathrm{k}$ & $13(37.1 \%)$ \\
$75-100 \mathrm{k}$ & $2(5.7 \%)$ \\
$>100 \mathrm{k}$ & $8(22.9 \%)$ \\
Marital status $(n, \%)$ & \\
Single, never married & $19(54.3 \%)$ \\
Married, living with partner & $12(34.3 \%)$ \\
Divorced, separated, widowed & $4(11.4 \%)$ \\
\hline
\end{tabular}

$\mathrm{HC}=$ healthy control participant; $n=$ number $\%=$ percentage; $M=$ mean; $S D=$ standard deviation; $\mathrm{k}=$ thousand

\section{Procedure}

Participants were recruited through community flyers and Web listings. After passing the initial telephone interview, online screener, and clinical diagnostic interview based on the Anxiety Disorders Interview Schedule for the DSM-IVLifetime version (Di Nardo, Brown, \& Barlow, 1994) to ensure no history of Axis I psychiatric disorders, participants were invited to enroll. We did not assess eating or personality disorders. After completing self-report inventories and demographics, each participant identified six distinct autobiographical situations characterized by social embarrassment, shame, or humiliation and composed a single paragraph and 10 negative self-beliefs for each of the situations. This material was used as stimuli for the autobiographical social situation task conducted in the MRI experiment approximately 1 week later.

\section{Autobiographical social situation task}

For each of the six personally salient, emotionally evocative autobiographical social situations, participants (a) wrote a single paragraph of up to 16 sentences to describe what happened, and (b) identified several situation-related negative self-beliefs that they held about themselves. Experimenters edited and entered the participant's content into an E-Prime script that presented each autobiographical situation as a series of eight 1-line sentences. The purpose of having participants read their own social situations was to help them recall the specific social context and prime more potent emotional reactivity to their own self-relevant negative self-beliefs. After reading the story sentences, nine negative self-beliefs were presented.

Before scanning, participants were briefly trained on the four conditions (neutral, react, reappraise, acceptance). Using experimenter-chosen stimuli, participants practiced reading neutral statements, reacting to negative self-beliefs by considering how it reflected something true about themselves, reappraisal of negative beliefs by using reappraisal to "actively reframe the belief by thinking in a way that reinterprets the content of the belief and thereby make the belief less negative and toxic for you," and acceptance of negative selfbeliefs by "simply observing and accepting without judgment from moment to moment (and not attempting to modify or change) any responses, including thoughts, emotions, memories, images, and physical sensations." Participants read written descriptions of react, reappraisal, and acceptance; verbalized their understanding to an experimenter; practiced implementing reappraisal and acceptance while verbalizing in real time what they were thinking and doing; and received feedback from the experimenter on their application of reappraisal and acceptance until there was clear demonstration of understanding of how to implement reappraisal and acceptance effectively (see Appendix). An example of acceptance 
might include observing the changing nature of thoughts, associations, memories, mental images, and physical sensations when interacting with each negative self-belief. For reappraisal, participants might encounter a negative self-belief, such as "NO ONE LIKES ME," and then be cued to reframe this belief by telling themselves, "That is not always true," "Some people like me," or "This is only a thought, not a fact."

During scanning, the experimental task consisted of three functional runs, each lasting 8 minutes and 33 seconds (see Fig. 1). Each run consisted of one neutral situation and two negative social situations (one with a reappraisal cue and one with a mindful attention cue). Each neutral situation consisted of six sentences presented for $3 \mathrm{~s}$ each (total $=18 \mathrm{~s}$ ), followed immediately by six neutral statements in uppercase boldface font presented for $12 \mathrm{~s}$ each. Each negative social situation consisted of participant-specific, personally salient autobiographical social situations as an unfolding story consisting of eight sentences presented for $3 \mathrm{~s}$ each (total $=24 \mathrm{~s}$ ). The story was immediately followed by nine trials of the participant's own situation-related negative self-beliefs. Each set of nine negative self-beliefs was independent and related to a specific autobiographical social situation. Trials 1, 3, and 5 were fixed as "REACT" cue trials, and the remaining six trials were fixed with one strategy only per social situation: either mindful attention cued with "OBSERVE" or reappraisal cued with "REFRAME."

After each trial, participants rated, using a button-response pad in their right hand, "How negative do you feel right now?" $(1=$ not at all to 5 = very much $)$. Each 18 -s trial consisted of negative self-belief $(0-3 \mathrm{~s})$, react, observe or reframe cue above negative self-belief (3-15 s), and emotion ratings $(15-18 \mathrm{~s})$. We examined only the $12 \mathrm{~s}$ of each trial during which the negative self-belief and the cue ("read," "react," "reframe," or "observe") were on the screen. Additionally, after each functional run, participants rated while inside the scanner perceived success when implementing the reappraisal and mindful attention strategies. They used the button-response pad and the scale of 1 (not at all) to 5 (very much) to make responses.

\section{Image acquisition}

We used a GE 3-T Signa magnet with a T2*-weighted gradient echo spiral-in/out pulse sequence (Glover \& Law, 2001) to acquire 1,026 functional volumes across three functional runs from 22 axial slices (interleaved bottom/up, frequency encoding $\mathrm{A} / \mathrm{P}$, repetition time $=1,500$ milliseconds, echo time $=28.5$ milliseconds, flip angle $=65^{\circ}$, field of view $=22 \mathrm{~cm}$, matrix $=64 \times 64$, resolution $=3.438 \mathrm{~mm}^{2}($ in-plane $) \times 4.5 \mathrm{~mm}$ (through-plane). Head movement was minimized using a bite bar and foam padding. High-resolution anatomical scans were acquired using fast spin-echo spoiled GRASS $\left(.8594^{2} \times 1.5\right.$ $\mathrm{mm}$; field of view $=22 \mathrm{~cm}$, frequency encoding $=256$ ).

\section{FMRI data processing and analysis}

Analysis of Functional NeuroImages (AFNI) software (Cox, 1996) was used to remove the first four time points; remove outliers; register, motion correct, spatially smooth $\left(4 \mathrm{~mm}^{3}\right.$ isotropic kernel), high-pass filter $(.011 \mathrm{~Hz})$, linear detrend, and convert into percentage signal change each functional run. No volumes demonstrated motion in the $x, y$, or $z$

3 stories per functional run:

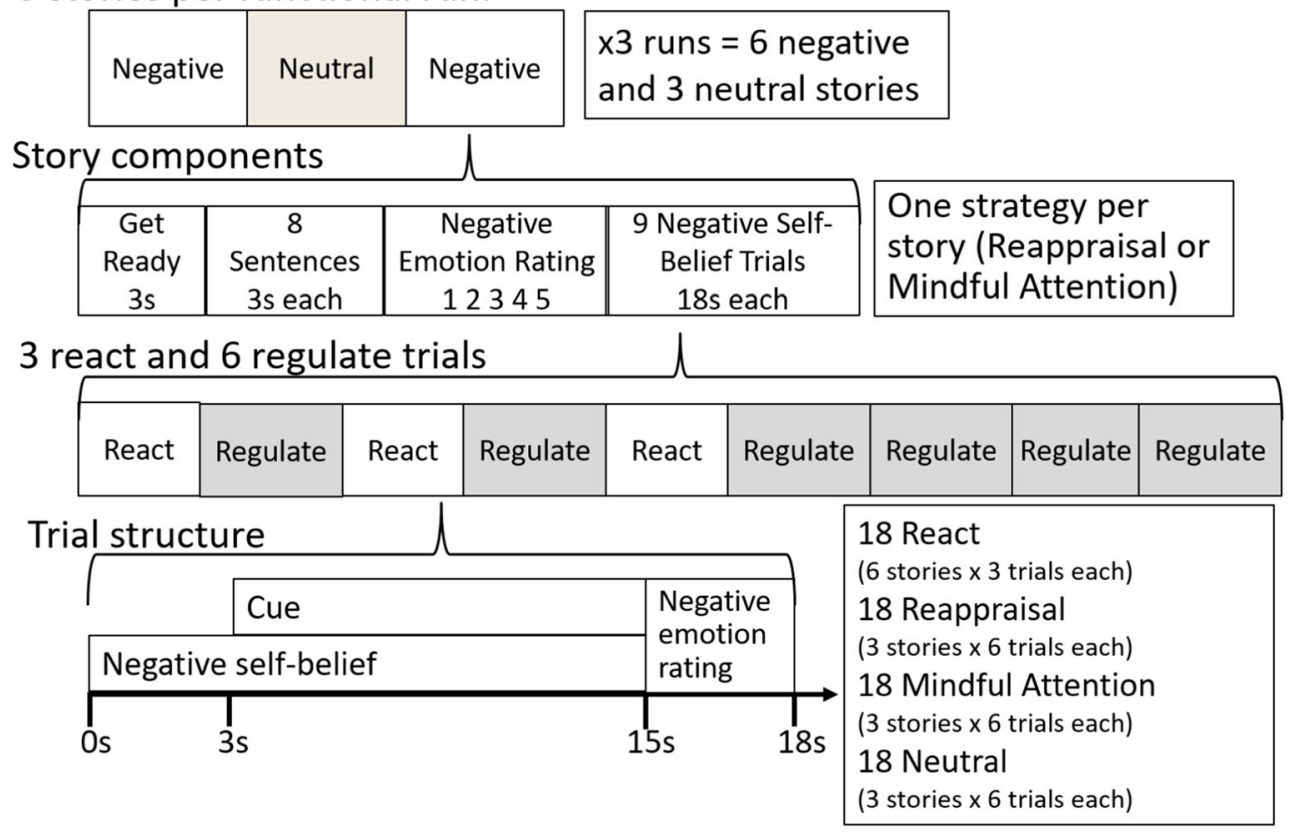

Fig. 1 Experimental task design 
directions in excess of $\pm 0.7 \mathrm{~mm}$. There was no evidence of stimulus-correlated motion (all $p$ s $>.38$ ).

We used 3 dDeconvolve to conduct a multipleregression that included removal of mean, linear, and quadratic trends, and motion-related variance in the BOLD signal. Regressors for neutral, react negative self-beliefs, reappraisal, and acceptance trials were convolved with the gamma variate model (Cohen, 1997) of the hemodynamic response function. Individual brain maps were resampled to $3.438-\mathrm{mm}$ cubic voxel, converted into Talairach atlas space (Talairach \& Tournoux, 1988), and second-level group statistical parametric maps were produced according to a random-effects model. To correct for multiple comparisons, we used 3dFWHMx to compute the intrinsic FWHM in $x, y, z$ directions and then $3 \mathrm{dClustSim}$ to determine the final joint probability threshold to protect against false-positive cluster detection (Forman et al., 1995). For 35 participants, $3 \mathrm{dClustSim}$ determined that the joint probability threshold of voxel-wise $p<.001$ and cluster volume $\geq 244$ $\mathrm{mm}^{3}\left(6\right.$ voxels $\left.\times 3.438 \mathrm{~mm}^{3}\right)$ resulted in false-positive cluster-wise protection at $p<.005$. Finally we used the FSL Randomize tool to implement threshold-free cluster enhancement (TFCE), which is a newer method for finding cluster-like structures that takes into account the spatial extent and height of BOLD signal and addresses problems with earlier versions of $3 \mathrm{dClust}$ (Smith \& Nichols, 2009). We confirmed the validity of the findings by reanalyzing the fMRI data using FSL software to implement preprocessing, statistical analysis, and TFCE. The results were identical.

For the amygdala analysis, we applied separate a priori left and right amygdala regions of interested (ROIs) maximum probability masks from the HarvardOxford subcortical brain atlas provided by the Harvard Center for Morphometric Analysis (http://harvard.eagle-i. net/sweet/provider?uri=http:/harvard.eagle-i.net/i/ 0000012e-9721-d0fd-55da-381e80000000) with a probability threshold at 0.50 . The regressor for each of the four conditions was convolved with the double gamma model of the hemodynamic response function. We computed the mean BOLD response per condition for the left and right amygdala ROI mask.

\section{Physiological data recording and analysis}

We measured concurrently with fMRI respiratory and cardiac data using the GE magnet built-in equipment and skin conductance with a BIOPAC system. The cardiac cycle was monitored using a photoplethysmogram placed on the right index finger. Skin conductance level was measured at 1,000 samples/second with two censors attached to the proximal phalanges of the second and fourth fingers on the left hand. Respiration was monitored using a bellows strapped around the upper thorax. A time stamp on the output permitted temporal registration to the BOLD functional images. After removing outliers, we computed mean values for respiration rate, heart rate, and skin conductance for each of the four conditions in the experimental task. We used a lag of $1.5 \mathrm{~s}$, equivalent to one TR in the fMRI time series, to capture autonomic responses. We used paired $t$ tests to investigate between-condition differences in each of the three autonomic variables.

\section{Results}

\section{Preliminary analyses}

As a manipulation check, we collected ratings inside the scanner immediately after each of the three functional runs. In Fig. 2 we show that participants reported no difference in perceived success of implementation of mindful attention between the three functional runs (all pairwise contrasts $p s>.32$ ), and of reappraisal between the three functional runs (all pairwise contrasts $p \mathrm{~s}>.11$ ). There was no Run $\times$ Regulation strategy interaction of perceived success when implementing regulation strategies, $F(2,33)=$ $0.90, p=.42$.

Paired $t$ tests showed that, compared with reading neutral statements, reacting to negative self-beliefs resulted in significantly greater self-reported negative emotion (Mean $\pm S D$; react vs. neutral: $3.19 \pm .81$ vs. $1.05 \pm .13), t(34)=15.09, p<.001,95 \%$ CI $[1.85$, 2.43] (see Fig. 3). Reacting to negative self-beliefs (vs. reading neutral statements) was also associated with greater respiration rate (react vs. neutral; $18.63 \pm 3.22$ vs. $17.90 \pm 3.05), t(34)=3.86, p<.001,95 \%$ CI $[.34$, 1.11], and heart rate (react vs. neutral; $68.49 \pm 9.69$ vs. $67.34 \pm 9.11), t(34)=4.18, p<.001,95 \%$ CI $[.57$, 1.66]. There was no differential skin conductance response (react vs. neutral; $.133 \pm .288$ vs. $.129 \pm .289$ ), $t(34)=.63, p=.53,95 \%$ CI $[-.007, .014]$ (see Fig. 4).

Neurally, compared with reading neutral statements, reacting to negative self-beliefs resulted in significantly greater BOLD responses in the frontopolar medial PFC, bilateral PCC, left angular gyrus, left amygdala, bilateral hippocampus, left caudate, and bilateral cerebellum, and lesser BOLD responses in the left DLPFC, bilateral lingual gyrus, right posterior middle temporal gyrus, and right inferior parietal lobule (see Table 2 and Fig. 5). The a priori amygdala ROI analysis showed greater BOLD responses for react negative self-beliefs versus neutral in left, $t(34)=3.33, p=.002$, and right amygdala, $t(34)=2.69, p=.011$ (see Fig. 6). 
5
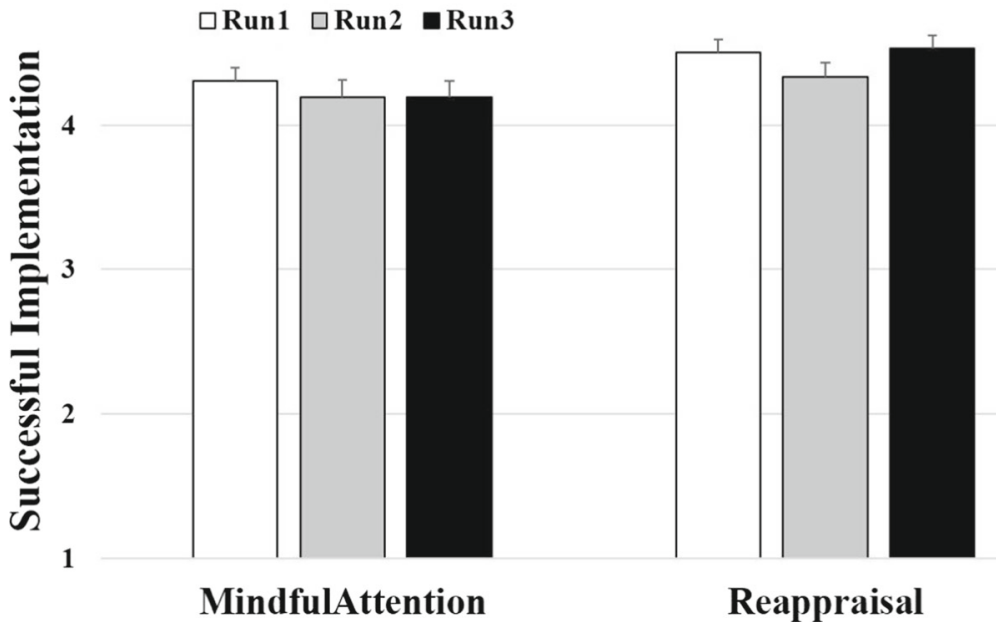

Error bars $=$ standard error of the mean

Fig. 2 Self-reported successful implementation of mindful attention and reappraisal after each functional run. Error bars = standard error of the mean

\section{Reappraisal}

Behavioral responses Compared with react to negative selfbeliefs, reappraisal resulted in significant lesser negative emotion $($ mean $=2.09 \pm .60$ vs. $3.19 \pm .81), t(34)=9.92, p$ $<.001,95 \%$ CI $[-1.33,-.88]$ (see Fig. 3).

Autonomic responses Compared with react, reappraisal resulted in significantly lesser respiration rate (Lag $1 \Delta=-.37, S D$ $=1.04$; reappraisal vs. react; $18.26 \pm 3.19$ vs. $18.63 \pm 3.22$ ), $t(34)=2.10, p<.05,95 \%$ CI $[-.73,-.01]$, but no difference for heart rate (Lag $1 \Delta=-.32, S D=1.10$; reappraisal vs. react; $68.17 \pm 9.45$ vs. $68.49 \pm 9.69), t(34)=1.71, p=.10,95 \% \mathrm{CI}$ $[-.70, .06]$, and skin conductance level $($ Lag $1 \Delta=-.003, S D=$ .015 ; reappraisal vs. react; $.129 \pm .289$ vs. $.133 \pm .288), t(34)=$ $1.24, p=.22,95 \%$ CI $[-.008, .002]$ (see Fig. 4).

Whole-brain responses Compared with react, reappraisal resulted in significantly greater BOLD responses in the DMPFC, left DLPFC, VLPFC, anterior temporal pole, posterior middle temporal gyrus, medial cuneus, and caudate, and right fusiform gyrus, caudate, anterior temporal

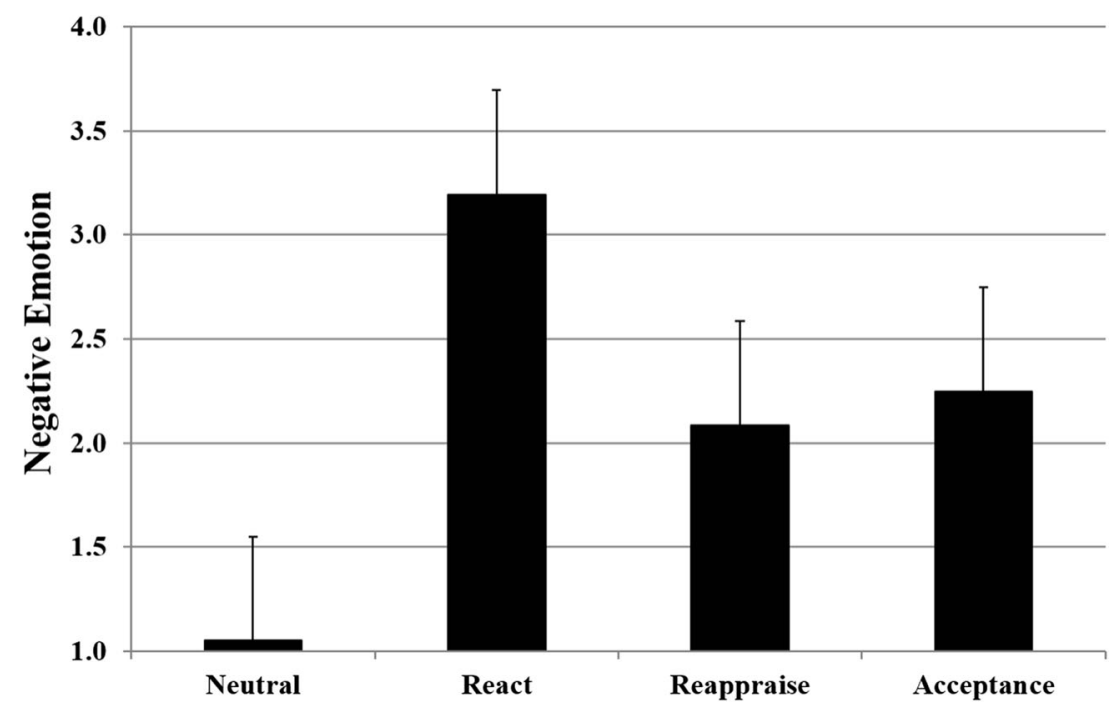

Fig. 3 Negative emotion ratings. Note. Negative emotion ratings range from 1 (not negative) to 3 (moderately negative) to 5 (very negative). Neutral $=$ reading neutral statements; react $=$ reacting to idiographic down-regulate reactivity to negative self-beliefs; acceptance $=$ implementing acceptance perspective toward reactivity to negative selfbeliefs. Error bars $=$ standard error of the mean 
a Respiration Rate

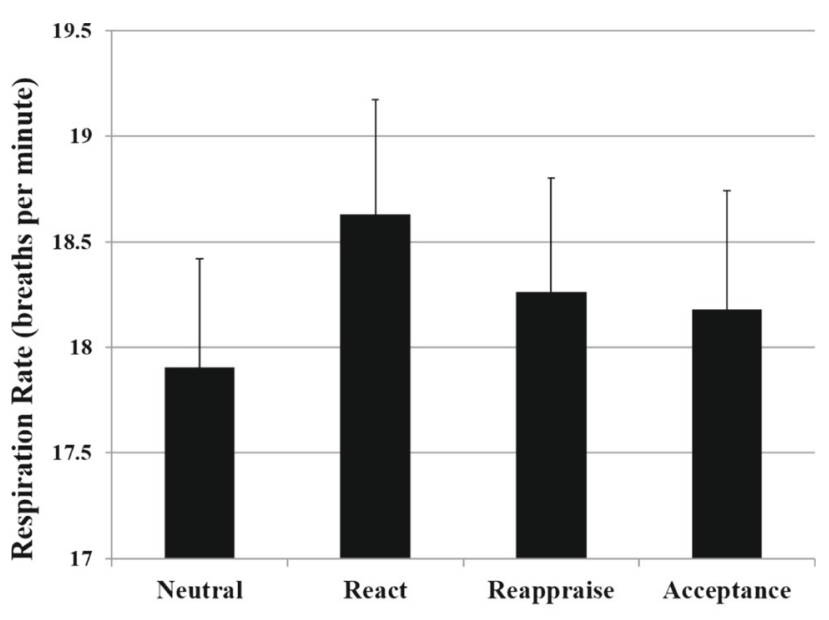

b Heart Rate

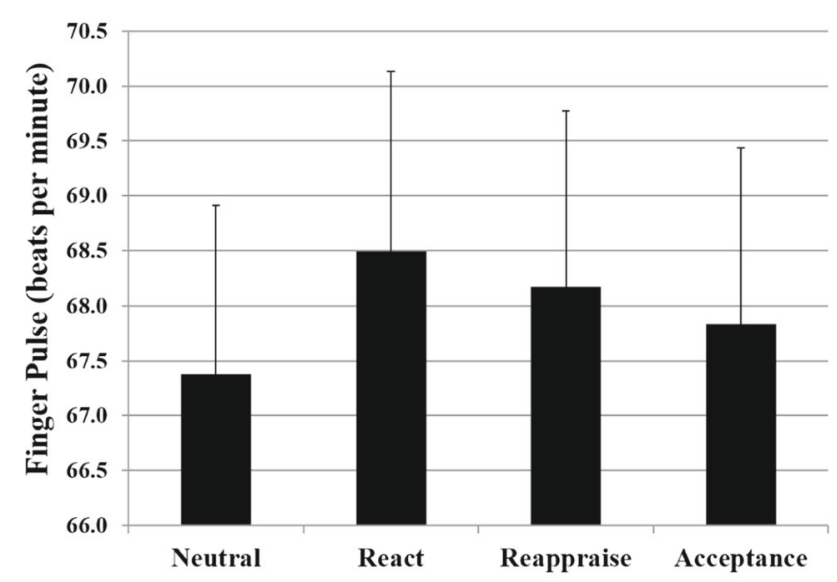

C Skin Conductance Level

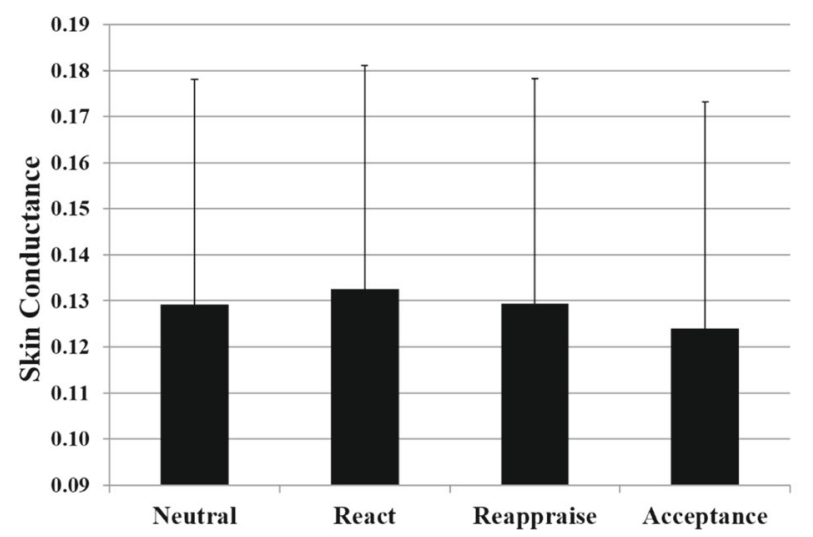

Error bars $=$ standard error of the mean.

Fig. 4 a Respiration rate, $\mathbf{b}$ heart rate, and $\mathbf{c}$ skin conductance level. Error bars $=$ standard error of the mean

pole, precentral cortex, as well as lesser BOLD response in the medial precuneus (see Fig. 7 and Table 3).
Amygdala ROI Compared with react, reappraisal resulted in significant decreases in BOLD signal in the left amygdala (mean difference $=-.17, S D=.19,95 \% \mathrm{CI}$ $[-.24,-.11]), t(34)=5.49, p<.001$, and right amygdala (mean difference $=-.14, S D=.17,95 \%$ CI $[-.20$, $-.08]$ ), $t(34)=4.68, p<.001$ (see Fig. 6) ROIs.

\section{Acceptance}

Behavioral responses Compared with react, acceptance resulted in significantly lesser negative emotion (mean $=2.25 \pm .63$ vs. $3.19 \pm .81), t(34)=8.34, p<.001,95 \%$ CI $[-1.17,-.72]$ (see Fig. 3).

Autonomic responses Compared with react, acceptance resulted in significantly lesser respiration rate (Lag $1 \Delta=-.45, S D$ $=1.07$; acceptance vs. react; $18.18 \pm 3.33$ vs. $18.63 \pm 3.22$ ), $t(34)=2.50, p=.017,95 \%$ CI $[-.82,-.08]$, and heart rate (Lag $1 \Delta=-.66, S D=1.05$; acceptance vs. react; $67.84 \pm 9.48$ vs. $68.49 \pm 9.69), t(34)=3.68, p=.001,95 \% \mathrm{CI}[-1.02,-.29]$, but no difference in skin conductance level (Lag $1 \Delta=-.009$, $S D=.030$; acceptance vs. react; $.124 \pm .291$ vs. $.133 \pm .288)$ $t(34)=1.68, p=.10,95 \%$ CI $[-.019, .002]$ (see Fig. 4).

Whole-brain responses Compared to react, acceptance resulted in significantly greater BOLD responses in the medial PFC, DLPFC, VLPFC, caudate, and bilateral inferior parietal lobule and caudate, as well as lesser BOLD responses in the left supramarginal gyrus and right cerebellar culmen (see Fig. 8 and Table 4).

Amygdala ROI Compared to react, acceptance did not result in significant decreases in BOLD signal in the left amygdala (mean difference $=-.04, S D=.13,95 \%$ CI $[-.09, .00]$ ), $t(34)=1.81, p=.088$, and right amygdala (mean difference $=-.035, S D=.13,95 \% \mathrm{CI}[-.08, .01]), t(34)=1.64, p=.12$ (see Fig. 6).

\section{Acceptance versus reappraisal}

Behavioral responses Compared with acceptance, reappraisal resulted in significantly greater reduction of negative emotion (mean acceptance $=2.25 \pm .63$ vs. reappraisal $=2.09 \pm .60$ ), $t(34)=2.27, p=.03,95 \%$ CI $[-.31,-.02]$ (see Fig. 3).

Autonomic responses Compared with acceptance, reappraisal resulted in no significant difference in respiration rate (Lag 1 $\Delta=.08, S D=.78$; reappraisal vs. acceptance; $18.26 \pm 3.19$ vs. $18.18 \pm 3.33), t(34)=.63, p=.54,95 \%$ CI $[-.18, .35]$, but led to a higher heart rate (Lag $1 \Delta=-.34, S D=.78$; reappraisal vs. acceptance; $68.17 \pm 9.45$ vs. $67.84 \pm 9.49), t(34)=2.57, p$ $=.015,95 \%$ CI [-.07, -.61], and marginally higher skin conductance level (Lag $1 \Delta=-.005, S D=.016$; reappraisal vs. 
Table 2 FMRI BOLD responses for react negative self-beliefs versus neutral statements

\begin{tabular}{|c|c|c|c|c|c|c|}
\hline Brain region & BA & Peak $x, y, z$ (TT) & Peak voxel $Z$ & Cluster vol $\left(\mathrm{mm}^{3}\right)$ & Cluster $Z$ mean & Cluster $Z S D$ \\
\hline \multicolumn{7}{|l|}{ React $>$ Neutral } \\
\hline \multicolumn{7}{|l|}{ Frontal lobe } \\
\hline $\mathrm{R}$ precentral gyrus, dorsal anterior cingulate cortex & 6,32 & $34,-11,28$ & 5.28 & 14,886 & 3.51 & 0.52 \\
\hline $\mathrm{L}$ anterior cingulate cortex & 32 & $-20,24,31$ & 5.65 & 23,443 & 3.57 & 0.59 \\
\hline \multicolumn{7}{|l|}{ Temporal lobe } \\
\hline L superior temporal gyrus, caudate Tail & 22 & $-31,-51,14$ & 7.30 & 21,397 & 3.90 & 0.85 \\
\hline L middle temporal gyrus & 39 & $-44,-73,25$ & 4.07 & 1,029 & 3.31 & 0.34 \\
\hline \multicolumn{7}{|l|}{ Parietal lobe } \\
\hline $\mathrm{R}$ posterior cingulate cortex, caudate tail & 29 & $17,-41,14$ & 5.69 & 13,273 & 3.75 & 0.68 \\
\hline \multicolumn{7}{|l|}{ Occipital lobe } \\
\hline L fusiform gyrus / inferior occipital gyrus & 18 & $-25,-90,-14$ & 4.07 & 880 & 3.20 & 0.31 \\
\hline $\mathrm{R}$ lingual gyrus & 18 & $25,-99,-7$ & 3.88 & 496 & 3.18 & 0.27 \\
\hline \multicolumn{7}{|l|}{ Subcortical } \\
\hline $\mathrm{R}$ cerebellum tonsil, inferior semi-lunar lobule & & $51,-62,-41$ & 5.06 & 5,973 & 3.25 & 0.33 \\
\hline L cerebellum inferior semilunar lobule & & $-20,-72,-38$ & 4.30 & 1,294 & 3.18 & 0.30 \\
\hline $\mathrm{R}$ cerebellum uvula & & $14,-89,-24$ & 3.30 & 633 & 2.93 & 0.10 \\
\hline \multicolumn{7}{|l|}{ Neutral > React } \\
\hline \multicolumn{7}{|l|}{ Frontal lobe } \\
\hline R middle frontal gyrus, precentral gyrus & 8,9 & $42,7,41$ & 3.80 & 2,161 & 3.10 & 0.22 \\
\hline R middle frontal gyrus, superior frontal gyrus & 9,10 & $48,44,21$ & 3.78 & 1,710 & 3.09 & 0.23 \\
\hline L precentral gyrus & 6 & $-28,-7,48$ & 4.01 & 755 & 3.15 & 0.24 \\
\hline $\mathrm{L}$ inferior frontal gyrus, precentral gyrus & 6,9 & $-42,3,28$ & 4.53 & 2,981 & 3.31 & 0.38 \\
\hline \multicolumn{7}{|l|}{ Parietal lobe } \\
\hline $\mathrm{L}$ inferior parietal lobule & 40 & $45,-38,38$ & 3.65 & 2,386 & 3.00 & 0.17 \\
\hline $\mathrm{R}$ precuneus, inferior parietal lobule & 31,40 & $31,-69,21$ & 4.54 & 6,257 & 3.14 & 0.27 \\
\hline \multicolumn{7}{|l|}{ Occipital lobe } \\
\hline L precuneus, middle occipital gyrus & 19,31 & $-27,68,24$ & 3.74 & 1,125 & 3.06 & 0.19 \\
\hline \multicolumn{7}{|l|}{ Subcortical } \\
\hline R cerebellum culmen, lingual gyrus & 18 & $10,-69,-10$ & 4.90 & 5,975 & 3.30 & 0.43 \\
\hline L cerebellum declive & & $-24,-69,-14$ & 6.18 & 2,058 & 3.58 & 0.64 \\
\hline
\end{tabular}

\# = cluster number; $\mathrm{BA}=$ Brodmann area; $x, y, z=$ Talairach coordinates at peak blood oxygen level-dependent signal intensity voxel; Vol = volume in $\mathrm{mm}^{3} ; t$ value $\geq 3.59$; voxel $p<.001$; minimum cluster volume threshold $\geq 488 \mathrm{~mm}^{3}\left(6\right.$ voxels $\left.\times 3.438 \mathrm{~mm}^{3}\right)$; cluster $p<.005 ; \mathrm{L}=$ left; $\mathrm{R}=$ right; $\mathrm{PFC}=$ prefrontal cortex

acceptance; $.129 \pm .289$ vs. $.124 \pm .291), t(34)=1.98, p=$ $.056,95 \%$ CI $[.000, .011]$ (see Fig. 4).

Whole-brain responses Compared with reappraisal, acceptance resulted in no significantly greater BOLD responses (see Table 5). However, compared with acceptance, reappraisal produced greater BOLD responses in the DMPFC, left posterior middle temporal gyrus, bilateral fusiform gyrus, left inferior parietal lobule, right inferior and middle occipital gyrus, and cerebellum.

Amygdala ROI Compared with acceptance, reappraisal significantly reduced BOLD responses in the left amygdala (mean difference $=-.14, S D=.19,95 \%$ CI $[-.08,-.21]$ ), $t(34)=4.15, p<.001$, and right amygdala (mean difference
$=-.10, S D=.19,95 \%$ CI $[-.04,-.18]), t(34)=3.15, p=$ .003 (see Fig. 6).

\section{Discussion}

The focus of this study was to examine the differential impact of two distinct emotion regulation strategies - cognitive reappraisal and acceptance - in the context of reacting to idiographic negative self-beliefs embedded in autobiographical narratives. We used three different types of indicators of emotion reactivity, including self-reported negative emotion ratings, autonomic psychophysiology (respiration rate, heart rate, skin conductance level), and fMRI BOLD responses in 35 healthy adults. We found converging evidence of 


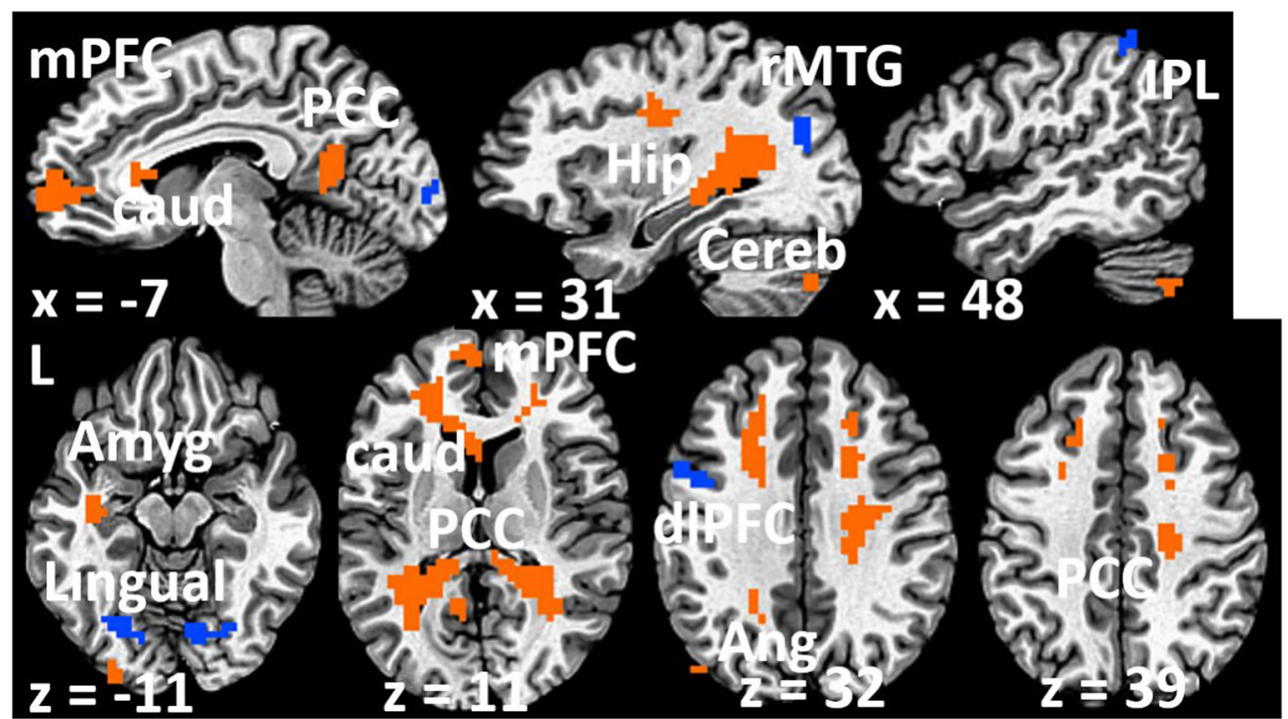

Fig. 5 FMRI BOLD responses for reacting to negative self-beliefs versus reading neutral statements. Note. $\mathrm{mPFC}=$ medial prefrontal cortex; $\mathrm{PCC}$ $=$ posterior cingulate cortex ; caud $=$ cuaduate rMTG $=$ right middle temporal gyrus; Hip = hippocampus; cereb $=$ cerebellum; IPL $=$ inferior parietal lobule; L = left; Amyg = amygdala; Lingual = lingual gyrus;

heightened emotional reactivity to negative self-beliefs reflected in all three indicators. Reappraisal and acceptance both produced down-regulation of negative emotion and autonomic measures and up-regulated activation of fMRI BOLD responses in regulatory brain networks. However, compared with acceptance, reappraisal resulted in greater reduction of negative emotion, less reduction in heart rate, greater overall cortical brain responses, and greater reduction in amygdala responses.

\section{Reactivity to negative self-beliefs}

As expected, compared with reading neutral statements, reacting to negative self-beliefs resulted in significantly greater emotion reactivity across self-report (negative emotion experience), autonomic psychophysiology (respiration rate and heart rate), and fMRI BOLD responses. Extensive research shows that in response to negative stimuli and emotions, a common pattern is increases in respiration rate via hyperventilation (Zvolensky \& Eifert, 2001) and heart rate (Brosschot \& Thayer, 2003). The observed brain regions associated with reacting to negative self-beliefs are implicated in emotion generation - for example, amygdala (LeDoux, 2007), emotional memory and episodic memory (PCC and hippocampus; Touroutoglou, Andreano, Barrett, \& Dickerson, 2015), and an anterior pole PFC region related to explicit processing and introspective evaluation of one's own thoughts and feelings (Christoff \& Gabrieli, 2000). The a priori amygdala ROI analysis confirmed greater left and right amygdala BOLD responses when reacting to negative self-beliefs versus reading neutral statements. These results confirm that
$\mathrm{dlPFC}=$ dorsolateral prefrontal cortex Ang $=$ angular gyrus. Orange $=$ greater BOLD signal for reacting to negative self-beliefs (vs neutral statements) Blue $=$ greater BOLD signal for reading neutral statements (vs reacting to negative self-beliefs). (Color figure online)

the negative self-beliefs were indeed inducing heightened negative emotion reactivity that would serve as a target for downregulation via the emotion regulation strategies.

\section{Cognitive reappraisal}

When compared with reacting, reappraisal of negative selfbeliefs resulted in significant decreases in self-reported negative emotion, respiration rate, a priori left and right amygdala ROIs, and increases in reappraisal-related prefrontal cortical cognitive control brain regions (Buhle et al., 2013). These findings demonstrate that reappraisal effectively down-regulated experiential, autonomic, and neural indicators of emotional reactivity.

These findings align with prior meta-analyses and reviews of fMRI of cognitive reappraisal to down-regulate negative emotional reactivity (Buhle et al., 2013; Ochsner et al., 2012). Specifically, the fMRI BOLD reappraisal activation of cognitive control (DMPFC, DLPFC, VLPFC) and linguistic processing (left VLPFC, left posterior middle temporal gyrus) brain regions directly replicate prior fMRI findings in a different sample of adults implementing reappraisal of idiographic negative self-beliefs embedded autobiographical narratives (P. R. Goldin et al., 2009). Furthermore, that reappraisal reduced elevated amygdala responses to negative selfbeliefs converges with our prior finding in healthy adults using a similar experimental design (P. R. Goldin et al., 2009). What is distinctive here is the coactivation of bilateral anterior temporal pole brain regions that are implicated in social cognition processing, specifically, representing and retrieving social knowledge (Olson, McCoy, Klobusicky, \& Ross, 2013). Thus, there is strong evidence of convergence 


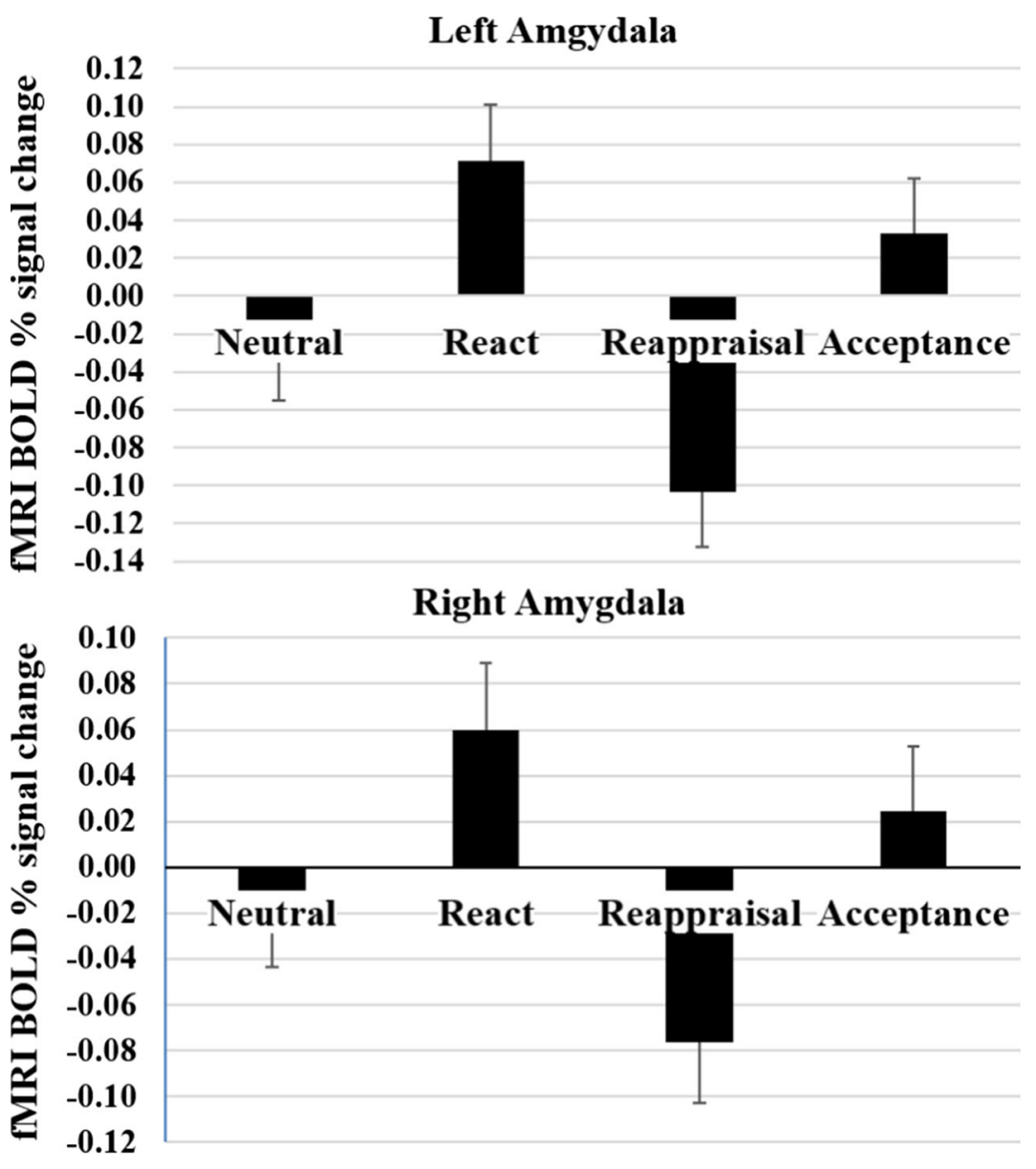

Error bars $=$ standard error of the mean.

Fig. 6 A priori anatomical amygdala region of interest fMRI BOLD signal responses. Error bars $=$ standard error of the mean

with reappraisal brain networks and reappraisal downregulation of negative emotions in the specific context of reacting to negative self-beliefs embedded in autobiographical narratives.

With regard to modulation of autonomic psychophysiological measures, reappraisal-related respiration rate has been reported in the context of using reappraisal to up-regulate and down-regulate responses to amusement (Giuliani, McRae, \& Gross, 2008). Our findings of decreases in respiration rate during reappraisal versus reacting to negative self-beliefs may reflect decreased emotional reactivity and/or increased focused attention during reappraisal (Stange, Hamilton, Fresco, \& Alloy, 2017). Interestingly, there were no differences between reappraisal and reacting to negative selfbeliefs in heart rate and skin conductance. This suggests that reacting and reappraising both involve sympathetic nervous system arousal when engaged in effortful processing of one's own negative self-beliefs. The reappraisal related modulation of heart rate is supported by a recent meta-analysis that found a modest but significant relationship between greater heart- rate variability and better top-down self-regulation, including emotion regulation (Holzman \& Bridgett, 2017).

\section{Acceptance}

Compared with reacting, acceptance of emotional reactivity to negative self-beliefs resulted in significant decreases in selfreported negative emotion, respiration rate, and heart rate; no changes in the left and right amygdala ROIs; and increases in a distributed network of prefrontal cortex brain regions implicated in cognitive control (medial PFC, DLPFC, VLPFC) and dorsal attention regulation (bilateral inferior parietal lobule regions that included the angular and supramarginal gyrus). The recruitment of multiple PFC regions during acceptance converges with a prior study of acceptance of emotional reactivity to sad films in healthy adults (Smoski et al., 2015), and acceptance toward personally salient worry statements in females with generalized anxiety disorder (Ellard et al., 2017). Reductions in respiration and heart rate have been reported in recent analyses of different meditation techniques (Gerritsen 


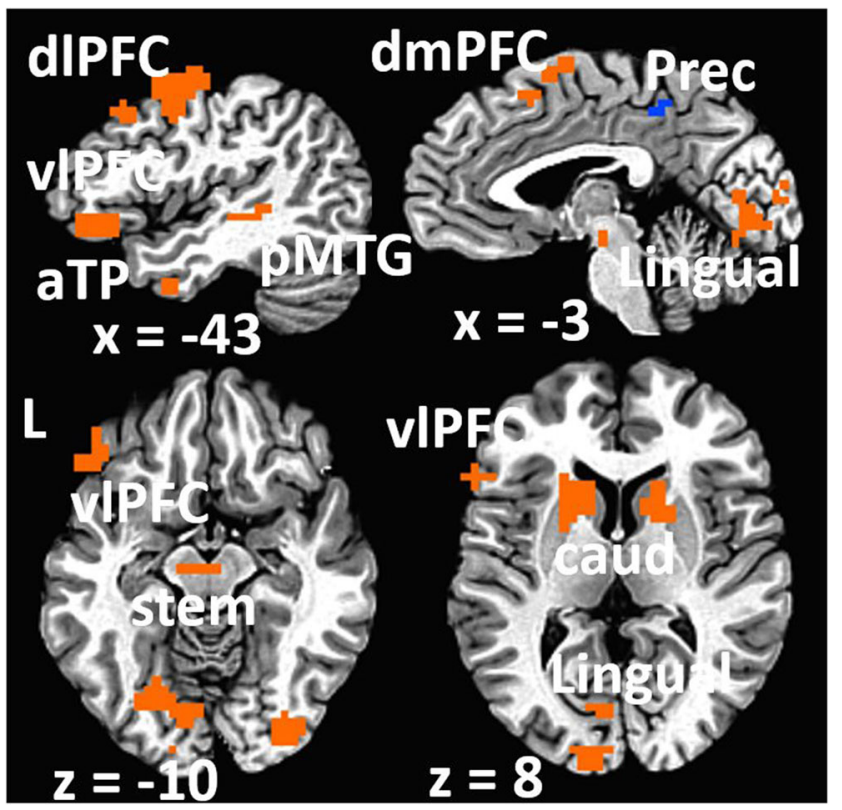

Fig. 7 FMRI BOLD responses for reappraisal versus reacting to negative self-beliefs. Note. dlPFC $=$ dorsolateral prefrontal cortex; vlPFC = ventrolateral prefrontal cortex; $\mathrm{pMTG}=$ posterior middle temporal gyrus; $\mathrm{aTP}=$ anterior temporal pole $\mathrm{dmPFC}=$ dorsomedial prefrontal

\& Band, 2018; Pascoe, Thompson, Jenkins, \& Ski, 2017) and with increases in cognitive and attentional engagement (Goosheh et al., 2017). The respiratory vagal nerve stimulation model identifies direct and indirect pathways by which cognition, affect, respiration, and heart rate interact (Gerritsen \& Band, 2018). Thus, this pattern of results suggests that acceptance down-regulated experiential and autonomic indicators of emotional reactivity while recruiting cognitive and attention regulatory brain networks.

\section{Reappraisal versus acceptance}

The direct contrast of reappraisal versus acceptance demonstrated that, compared with acceptance, reappraisal resulted in significantly lesser self-reported negative emotion and bilateral amygdala responses, as well as greater heart rate and frontal-temporal-parietal-occipital brain activation. The cooccurrence of increased medial PFC recruitment, especially the dorsal anterior cingulate and dorsomedial prefrontal cortex, with decreased amygdala responses, converges with prior evidence of an inverse functional connectivity during reappraisal of negative emotion in general (Banks, Eddy, Angstadt, Nathan, \& Luan Phan, 2007; Ochsner et al., 2012), and specifically when reappraising negative selfbeliefs (P. R. Goldin et al., 2009). The absence of any brain regions that were more activated during acceptance than during reappraisal suggests that the acceptance strategy may entail lesser cognitive processing, specifically in the context of reacting to negative self-beliefs. The interpretation that acceptance-based coping with negative emotions is more cortex Prec $=$ precuneus; Lingual $=$ lingual gyrus; $\mathrm{L}=$ left $;$ stem $=$ brain stem; caud $=$ cuaduate orange $=$ greater BOLD signal for reappraisal vs . reacting to negative self-beliefs blue $=$ greater $\mathrm{BOLD}$ signal for reacting vs. reappraisal of negative self-beliefs. (Color figure online)

efficient than other emotion-regulation strategies has been reported previously when acceptance was compared with expressive suppression (Alberts, Schneider, \& Martijn, 2012). In summary, this pattern of results suggests that reappraisal was more successful than acceptance at reducing emotional reactivity with more effortful processing as indicated by significantly greater autonomic activation (i.e., heart rate) and mental engagement (i.e., overall greater brain activation).

Neurally, acceptance and reappraisal produced similar activation of dorsomedial PFC, bilateral VLPFC, and DLPFC regions implicated in functional components of cognitive reappraisal of emotion, including selecting a specific strategy, implementing that strategy, and monitoring its application (Buhle et al., 2013). Effective connectivity analyses have shown that these dorsal and inferior PFC regions are strongly interconnected and likely reflect the interaction of working memory and goal-appropriate emotion regulation strategy selection to shape cognition and achieve a desired behavior (in this case, reduction of emotional reactivity; Morawetz, Bode, Baudewig, Kirilina, \& Heekeren, 2016). The right VLPFC is thought to be critical to various control processes that are essential for goal-oriented cognition. Relevant to both acceptance and reappraisal, specific subcomponents of the right VLPFC are involved in overriding a prepotent response tendency, reflexive reorienting, and updating of action plans (Levy \& Wagner, 2011). Specifically, the ability to stop automatic habits of mind and redirect attention and behavior to respond in a more skillful manner is a fundamental component of both acceptance and reappraisal emotion-regulation strategies. The right VLPFC has been identified as an important 
Table 3 FMRI BOLD responses for cognitive reappraisal versus react negative self-beliefs

\begin{tabular}{|c|c|c|c|c|c|c|}
\hline Brain region & BA & Peak $x, y, z(\mathrm{TT})$ & Peak voxel $Z$ & Cluster vol $\left(\mathrm{mm}^{3}\right)$ & Cluster $Z$ mean & Cluster $Z S D$ \\
\hline \multicolumn{7}{|l|}{ Reappraisal > React } \\
\hline \multicolumn{7}{|l|}{ Frontal lobe } \\
\hline L middle / inferior frontal gyrus & 8,45 & $-38,21,41$ & 5.53 & 14,716 & 3.36 & 0.45 \\
\hline $\mathrm{R}$ inferior frontal gyrus & 47 & $48,31,-10$ & 3.81 & 719 & 3.05 & 0.19 \\
\hline L superior frontal gyrus & 9 & $-14,55,41$ & 3.96 & 1,127 & 3.10 & 0.24 \\
\hline $\mathrm{L}$ precentral gyrus & 6 & $-45,-3,58$ & 6.13 & 11,667 & 3.69 & 0.70 \\
\hline $\mathrm{R}$ medial frontal / precentral gyrus & 6 & $18,-10,55$ & 3.88 & 2,213 & 3.09 & 0.21 \\
\hline \multicolumn{7}{|l|}{ Temporal lobe } \\
\hline $\mathrm{L}$ anterior temporal pole & 21 & $-44,0,-31$ & 4.40 & 2,205 & 3.18 & 0.28 \\
\hline $\mathrm{R}$ anterior temporal pole & 38 & $55,10,-17$ & 4.79 & 757 & 3.37 & 0.42 \\
\hline L middle temporal gyrus & 22 & $-51,-38,0$ & 5.18 & 4,258 & 3.55 & 0.52 \\
\hline \multicolumn{7}{|l|}{ Parietal lobe } \\
\hline $\mathrm{R}$ postcentral / precentral gyrus & 3,4 & $62,-17,35$ & 4.05 & 1,461 & 3.14 & 0.26 \\
\hline \multicolumn{7}{|l|}{ Occipital lobe } \\
\hline L cuneus, lingual & 17,18 & $-7,-93,7$ & 5.64 & 33,097 & 3.37 & 0.45 \\
\hline \multicolumn{7}{|l|}{ Subcortical } \\
\hline L caudate & & $-17,0,18$ & 6.69 & 15,766 & 3.81 & 0.83 \\
\hline $\mathrm{R}$ caudate & & $17,3,20$ & 6.35 & 7,577 & 3.66 & 0.64 \\
\hline L midbrain & & $-10,-17,-13$ & 5.27 & 899 & 3.36 & 0.48 \\
\hline \multicolumn{7}{|l|}{ React $>$ Reappraisal } \\
\hline \multicolumn{7}{|l|}{ Occipital lobe } \\
\hline Medial precuneus & 7 & $0,-41,41$ & 4.37 & 1,474 & 3.28 & 0.36 \\
\hline
\end{tabular}

\# = cluster number; $\mathrm{BA}=$ Brodmann area; $\mathrm{x}, \mathrm{y}, \mathrm{z}=$ Talairach coordinates at peak blood oxygen level-dependent signal intensity voxel; Vol = volume in $\mathrm{mm}^{3} ; t$ value $\geq 3.59$; voxel $p<.001 ;$ minimum cluster volume threshold $\geq 244 \mathrm{~mm}^{3}\left(6\right.$ voxels $\left.\times 3.438 \mathrm{~mm}^{3}\right)$; cluster $p<.005 ; \mathrm{L}=$ left; $\mathrm{R}=$ right; $\mathrm{PFC}=$ prefrontal cortex

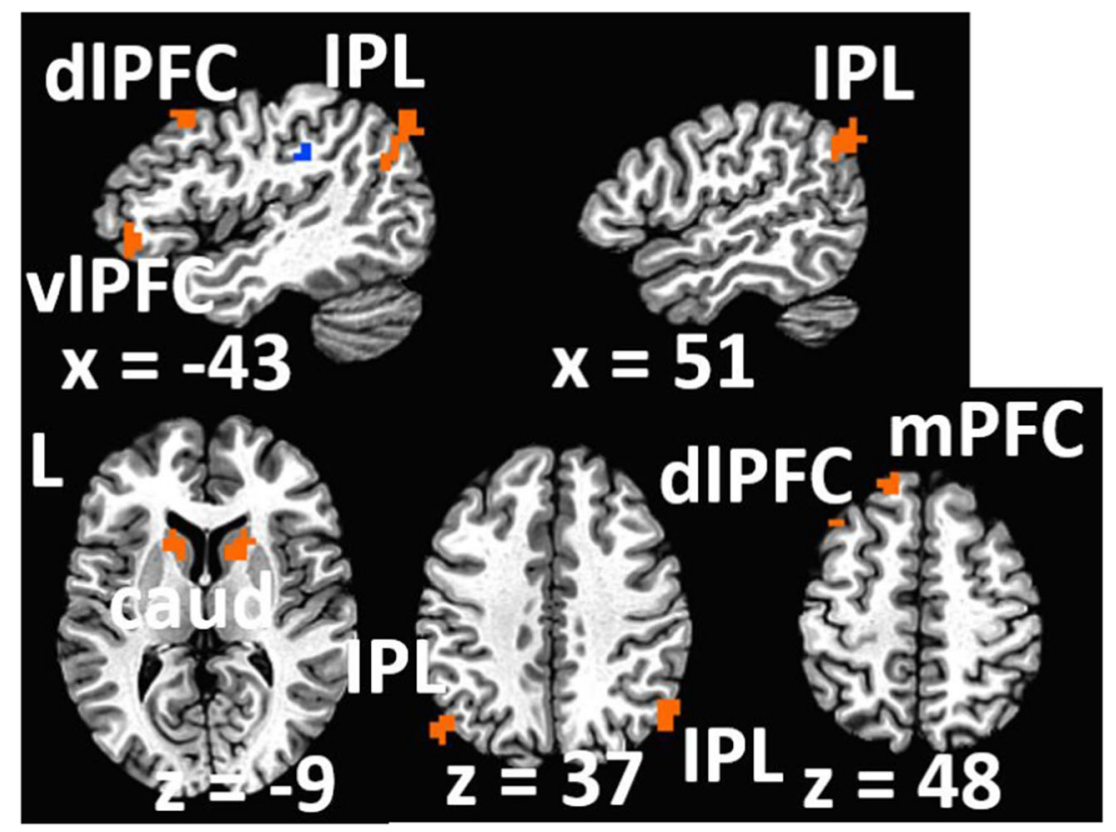

Fig. 8 FMRI BOLD responses for mindful attention versus reacting to negative self-beliefs. Note. dlPFC $=$ dorsolateral prefrontal cortex; IPL $=$ inferior parietal lobule; vlPFC = ventrolateral prefrontal cortex; $\mathrm{L}=$ left; caud $=$ cuaduate $; \mathrm{mPFC}=$ medial prefrontal cortex orange $=$ greater
BOLD signal for reappraisal vs. reacting to negative self-beliefs blue = greater BOLD signal for reacting vs. reappraisal of negative self-beliefs (Color figure online) 
Table 4 FMRI BOLD responses for acceptance versus reacting to negative Self-beliefs

\begin{tabular}{|c|c|c|c|c|c|c|}
\hline Brain region & $\mathrm{BA}$ & Peak $x, y, z(\mathrm{TT})$ & Peak voxel $Z$ & Cluster vol $\left(\mathrm{mm}^{3}\right)$ & Cluster $Z$ mean & Cluster $Z S D$ \\
\hline \multicolumn{7}{|l|}{ Acceptance $>$ React } \\
\hline \multicolumn{7}{|l|}{ Frontal lobe } \\
\hline L superior frontal gyrus & 8 & $-10,41,48$ & 3.95 & 2,513 & 3.16 & 0.24 \\
\hline $\mathrm{R}$ superior frontal gyrus & 8 & $17,41,41$ & 3.76 & 613 & 3.05 & 0.19 \\
\hline L inferior frontal gyrus & 10 & $-41,5,10$ & 3.21 & 542 & 2.94 & 0.09 \\
\hline L superior/middle frontal gyrus & 8 & $-38,21,44$ & 4.37 & 5,101 & 3.21 & 0.35 \\
\hline $\mathrm{L}$ inferior/middle frontal gyrus & 47 & $-45,38,-4$ & 4.65 & 3,090 & 3.23 & 0.34 \\
\hline $\mathrm{R}$ inferior/middle frontal gyrus & 47 & $41,37,-7$ & 3.86 & 624 & 3.07 & 0.21 \\
\hline \multicolumn{7}{|l|}{ Temporal lobe } \\
\hline L middle temporal gyrus & 21 & $-69,-28,-17$ & 3.80 & 526 & 3.01 & 0.18 \\
\hline \multicolumn{7}{|l|}{ Parietal lobe } \\
\hline $\mathrm{L}$ angular gyrus, precuneus, inferior parietal lobule & 19,39 & $-45,-76,38$ & 5.36 & 6,641 & 3.40 & 0.50 \\
\hline $\begin{array}{l}\mathrm{R} \text { angular/supramarginal gyrus, inferior parietal } \\
\text { lobule }\end{array}$ & 39,40 & $52,-62,34$ & 3.93 & 2,863 & 3.24 & 0.29 \\
\hline \multicolumn{7}{|l|}{ Subcortical } \\
\hline $\mathrm{R}$ caudate & & $11,10,11$ & 4.35 & 3,681 & 3.42 & 0.39 \\
\hline L caudate & & $-17,7,0$ & 4.52 & 3,484 & 3.32 & 0.37 \\
\hline \multicolumn{7}{|l|}{ React $>$ acceptance } \\
\hline \multicolumn{7}{|l|}{ Frontal lobe } \\
\hline $\mathrm{L}$ anterior cingulate & 32 & $-18,24,24$ & 5.09 & 1,491 & 3.53 & 0.52 \\
\hline \multicolumn{7}{|l|}{ Parietal lobe } \\
\hline L IPL, supramarginal gyrus & 40 & $-59,-34,26$ & 5.76 & 5,112 & 3.38 & 0.56 \\
\hline \multicolumn{7}{|l|}{ Subcortical } \\
\hline $\mathrm{R}$ cerebellum tonsil & & $31,-44,-31$ & 4.61 & 2,238 & 3.32 & 0.40 \\
\hline $\mathrm{R}$ poster cingulate / caudate tail & 31 & $21,-38,21$ & 4.40 & 1,476 & 3.28 & 0.37 \\
\hline
\end{tabular}

\# = cluster number; $\mathrm{BA}=$ Brodmann area; $\mathrm{x}, \mathrm{y}, \mathrm{z}=$ Talairach coordinates at peak blood oxygen level-dependent signal intensity voxel; Vol = volume in $\mathrm{mm}^{3}, t$ value $\geq 3.59$; voxel $p<.001$; minimum cluster volume threshold $\geq 244 \mathrm{~mm}^{3}\left(6\right.$ voxels $\left.\times 3.438 \mathrm{~mm}^{3}\right)$; cluster $p<.005 ; \mathrm{L}=$ left; $\mathrm{R}=$ right; $\mathrm{PFC}=$ prefrontal cortex

node in a right-hemisphere-dominant ventral frontoparietal network that supports reorienting (Corbetta, Patel, \& Shulman, 2008). Reorienting involves interrupting automatic response (e.g., distorted beliefs and interpretations), resetting ongoing cognitive processes (e.g., avoidance tendencies), and considering more adaptive ways of responding to threatening stimuli (e.g., negative self-beliefs) via recruitment of complementary brain networks such as the dorsal frontoparietal network specialized for selecting and linking stimuli and responses. Clearly, acceptance and reappraisal both rely on the specific use of reorienting to switch between multiple brain networks related to inhibitory control, attention allocation, and cognitive control. Thus, overlapping PFC recruitment suggests shared cognitive functions when implementing reappraisal and acceptance ER strategies.

However, acceptance also produced recruitment of the bilateral dorsal parietal (angular gyrus, IPL, intraparietal sulcus, supramarginal gyrus) regions that were not detected during reappraisal. Different regions within the parietal cortex are associated with ventral ("bottom-up") and dorsal ("top-down) attention networks (Corbetta \& Shulman, 2002). During acceptance, attention is volitionally orientated to moment-to-moment experience with no explicit intention to change one's response to emotional stimuli. Reappraisal, which is a cognitive change strategy, also involves volitional orienting of attention. The presence of bilateral dorsal parietal cortical activation during acceptance suggests top-down guided voluntary allocation of attention to features of emotion experience, which has generally been associated with the dorsal frontoparietal system. The dorsal parietal cortex regions recruited during acceptance in this study overlap with parietal brain activations detected during mindful attention regulation of reactivity to negative self-beliefs in adults with social anxiety disorder following 2 months of MBSR training (P. Goldin, Ziv, Jazaieri, Hahn, \& Gross, 2013). The mindful attention regulation strategy used in the mindfulness training study was described as cultivating a present moment, metacognitive attentional perspective that just notices any thought, memory, emotion or sensation. 
Table 5 FMRI BOLD responses for acceptance versus cognitive reappraisal of negative self-beliefs

\begin{tabular}{|c|c|c|c|c|c|c|}
\hline Brain region & BA & Peak $x, y, z$ (TT) & Peak voxel $Z$ & Cluster vol $\left(\mathrm{mm}^{3}\right)$ & Cluster $Z$ mean & Cluster $Z S D$ \\
\hline Acceptance $>$ Reappraisal & & none & & & & \\
\hline \multicolumn{7}{|l|}{ Reappraisal > Acceptance } \\
\hline \multicolumn{7}{|l|}{ Frontal lobe } \\
\hline $\mathrm{L}$ dorsal anterior cingulate / middle frontal gyrus & 32,9 & -172428 & 4.84 & 7,474 & 3.26 & 0.39 \\
\hline L precentral gyrus & 6 & $-55,-4,38$ & 3.82 & 1,996 & 3.09 & 0.21 \\
\hline \multicolumn{7}{|l|}{ Temporal lobe } \\
\hline L middle temporal gyrus & 21,22 & $-48,-31,0$ & 4.61 & 5,118 & 3.37 & 0.40 \\
\hline $\mathrm{R}$ middle temporal gyrus & 21 & $38,-31,0$ & 4.40 & 576 & 3.18 & 0.32 \\
\hline \multicolumn{7}{|l|}{ Parietal lobe } \\
\hline $\mathrm{L}$ inferior parietal lobule & 40 & $-38,-38,28$ & 4.21 & 885 & 3.21 & 0.30 \\
\hline \multicolumn{7}{|l|}{ Occipital lobe } \\
\hline $\begin{array}{l}\mathrm{L} \text { and } \mathrm{r} \text { cerebellar tonsil, lingual, fusiform gyrus, } \\
\text { mid occipital gyrus }\end{array}$ & 18,19 & $34,-52,-31$ & 5.15 & 48,844 & 3.20 & 0.32 \\
\hline \multicolumn{7}{|l|}{ Subcortical } \\
\hline $\mathrm{R}$ caudate & & $20,4,21$ & 4.29 & 1,863 & 3.09 & 0.28 \\
\hline
\end{tabular}

$\mathrm{BA}=$ Brodmann area; $x, y, z(\mathrm{TT})=$ Talairach coordinates at peak blood oxygen level-dependent signal intensity voxel; Vol $=$ volume in mm ${ }^{3} ;$ voxel $Z$ score $\geq 2.81$; voxel $p<.005$; minimum cluster volume threshold $\geq 488 \mathrm{~mm}^{3}\left(12\right.$ voxels $\left.\times 3.438 \mathrm{~mm}^{3}\right)$; cluster $p<.005 ; \mathrm{L}=$ left; $\mathrm{R}=$ right

Currently, there is only one fMRI study that directly compared reappraisal with acceptance (Smoski et al., 2015). Behaviorally, this study found greater decreases in selfreported negative emotion for reappraisal versus acceptance in both remitted depressed and healthy controls in the context of emotional reactivity to viewing sad images. Our study if 35 healthy adults produced the same result when reacting to negative self-beliefs. Neurally, the Smoski and colleagues study reported greater differential brain activation in left insula and right PFC during acceptance (vs. reappraisal), and greater activation in multiple PFC areas during reappraisal (vs. acceptance). The authors interpreted the neural findings as evidence that acceptance was more related to somatic and emotion awareness. However, the right PFC activation may reflect inhibitory control or other cognitive regulatory functions. In our study, no brain regions were more highly activated during acceptance compared with reappraisal. One interpretation of the disparity in results across these two studies is that reducing emotional reactivity to ecologically valid and personally salient stimuli (i.e., self-generated negative self-beliefs) required a higher level of effort for reappraisal of reactivity to sad images that were not idiographic. This suggests that the personal salience and emotional intensity of stimuli may interact with the degree of effort required to implement different emotion regulation strategies.

Another factor that might influence the ability to implement different emotion regulation strategies in the fMRI task context is the effect of timing of the cued regulation strategy and negative stimulus. In our study, the negative self-belief was presented for $3 \mathrm{~s}$ before the cue presentation. Thereafter, the belief and cue remained on the screen together for $12 \mathrm{~s}$.
Given the possibility of spontaneous emotion regulation, the precue 3-s window may have been associated with more automatic, less effortful emotion regulation compared with the subsequently cued emotion regulation 12 -s period. Furthermore, the time required to generate and implement reappraisal and mindful attention may vary. The timing may be influenced by individual differences in both the innate and trained ability to apply mindful attention and reappraisal. Given that reappraisal requires linguistic processing, appraisal, selection, and application of a specific reinterpretation, it may take more time to implement compared with mindful attention. Furthermore, prior regular use, self-efficacy, and learning history most likely affect patterns of emotional reactivity as well as the implementation of reappraisal and mindful attention in the fMRI task context.

\section{Limitations and future directions}

The current study investigated only two distinct theoretically defined emotion regulation strategies. It is important to note that, unlike reappraisal, the instructions for acceptance did not explicitly ask participants to intentionally and actively attempt to reduce reactivity to their own negative self-beliefs. This may have induced a demand effect in our study that favored reappraisal versus acceptance. However, by inhibiting automatic patterns of interpreting, elucidating, and reacting, mindful attention short-circuits overlearned mental habits, including emotion reactivity to perceived aversive stimuli such as negative self-beliefs. Future studies would benefit from comparing reappraisal and acceptance with other 
adaptive (e.g., problem-solving) and maladaptive (e.g., rumination, suppression) emotion-regulation strategies. Furthermore, these strategies were implemented in only one context: regulating reactivity to participantgenerated negative self-beliefs related to participantspecific autobiographical adverse social situations. Thus, although both strategies were effective in downregulating self-reported negative emotion reactivity, it is not clear how well these two strategies would function in other types of challenging contexts. Our study used a fixed sequence of a story followed by nine negative self-belief trials. To enhance reactivity, future studies may consider randomly inserting regulation of negative self-belief trials into the unfolding autobiographical narrative to decrease expectation effects and increase the uncertainty of stimulus presentation. Given the fMRI context and the recruitment of very healthy adults with no lifetime history of psychopathology, it is not clear how far the results of this one study can be generalized to others that include participants such as children/adolescents, older adults, and adults with psychopathologies such as mood and anxiety disorders. To examine the development of reappraisal and acceptance as ER strategies, it will be important for future studies to examine how mental training programs and clinical interventions modulate the brain and behavioral bases of reappraisal and acceptance.

Acknowledgments This research was supported by NIMH Grant R01 MH076074, awarded to James Gross. We wish to thank Gary Glover for his technical assistance with magnetic resonance imaging. Philippe Goldin, who is independent of any commercial funder, had full access to all of the data in the study and takes responsibility for the integrity of the data and the accuracy of the data analysis.

\section{Compliance with ethical standards}

Conflict of interest None of the authors of this manuscript have any biomedical financial interests or potential conflicts of interest.

\section{Appendix}

Autobiographical social situation task instructions

\section{General instructions}

You will be doing a task where you will be asked to read sentences that make up your own personal, autobiographical stories. You will be asked to react to these sentences how you naturally would, and you will also be asked to regulate your reaction. This study has very important implications for mental health and well-being. But it really requires your attention. Please stay focused, pay attention, and really engage with listening to the sentences.

\section{Read}

When you are given this prompt to READ, please read each statement that is presented on the screen.

Now, let's practice with some statements: "The ball is on the box."

Please explain back to me how you understand the READ instruction.

How confident do you feel, from 1 to10, that you can do this READ instruction to the sentences while you are in the scanner?

\section{React}

When you are given the prompt to REACT, as you read each statement, focus on how the statement is true for you. Allow yourself to REACT with the thoughts that you would actually have. So, this pine cone represents one of the critical sentences. Allow your body and mind to react as they naturally would to the statements.

Now, let's practice following the REACT instruction with some statements. "Others think you are awkward." Now, focus on when this is true for you - when people are uncomfortable being around you. Let one thought follow to the next. Stay immersed in your experience of knowing you are awkward. Stay in the first person and really think this is happening to you. Now, let's practice with another couple of sentences: "Others think you are uptight." Just let yourself

REACT as you normally would. Now, what was your experience of that?

Please explain back to me how you understand the REACT instruction.

How confident do you feel, from 1 to 10 , that you can do this REACT instruction to the sentences while you are in the scanner?

\section{Reappraisal}

For this part of the task, when you are given the prompt to REFRAME, we would like you to reinterpret the statement so it is less negative and toxic for you. We would like you to work with your thoughts so they change your sensations and feelings.

In contrast to our prickly reactivity, REFRAME is knowing that our thoughts, sensations, and experiences can be molded and shaped. It does not have to be rigid.

You will think of a short positive coping statement that directly REFRAMEs the thought to something more realistic. Often, what will work is to think of specific evidence against the thought. So, this is not to just "think positive" or tell yourself "It doesn't matter," as these are unrealistic and dismissive. But rather what is helpful is to come up with some evidence against your thought or think of another point of 
view - ones that you can buy into and believe. You really work with the thought and say it is not true.

So, if you read the sentence, "No one likes you," some REFRAMES to these sentence are:

"That is not true, some people like me," or "My sibling likes me."

Now, practice with these sentences:

"You are not very smart," or "People don't enjoy being around you."

Some REFRAMEs you can use with this are: "That is not always true"; "Some people like me"; "This is only a thought, not a fact."

Please explain back to me your understanding of the REFRAME instruction.

How confident do you feel, from 1 to 10 , that you can do this REFRAME instruction to the sentences while you are in the scanner?

\section{Acceptance}

When you are given the prompt OBSERVE, as you read each statement with this OBSERVE instruction, we'd like you to use the part of your mind that can notice your thoughts and sensations. As you read each statement, simply observe the sensations and thoughts that play out in your mind in reaction to this statement. Notice whether your thoughts arise as images, words, or both. Notice how different thoughts arise and fall and change over time naturally. Just notice, and watch what happens with a sense of curiosity about your experience as it unfolds. Just observe your thoughts, from moment to moment, without getting sucked into them.

So, let's practice now. Here are some sentences, and I'd like you to observe what thoughts you have in reaction to what I say.

Here is the first one:

"Others sometimes look down on you. You can be pitied by others."

Wait 10 seconds. What are some thoughts you had in reaction to those statements? Were you able to notice your thoughts?

Now, let's practice again.

"People seem to like you. You are a likeable person."

"Others look down on you. You are embarrassing."

"Do you have enough friends? You really don't have many friends."

"People are embarrassed to be seen with you. You are not liked."

Please explain back to me your understanding of the OBSERVE instruction.

How confident do you feel, from 1 to 10 , that you can do this OBSERVE instruction to the sentences while you are in the scanner?
Remember, with OBSERVE, you are curious, open, noticing, and fully experiencing without judgments, reactivity, or elaborating.

Publisher's note Springer Nature remains neutral with regard to jurisdictional claims in published maps and institutional affiliations.

\section{References}

Alberts, H. J. E. M., Schneider, F., \& Martijn, C. (2012). Dealing efficiently with emotions: Acceptance-based coping with negative emotions requires fewer resources than suppression. Cognition and Emotion, 26(5), 863-870. https://doi.org/10.1080/02699931.2011. 625402

Aldao, A., \& Nolen-Hoeksema, S. (2012). The influence of context on the implementation of adaptive emotion regulation strategies. Behaviour Research and Therapy, 50(7/8), 493-501. https://doi. org/10.1016/j.brat.2012.04.004

Aldao, A., Nolen-Hoeksema, S., \& Schweizer, S. (2010). Emotionregulation strategies across psychopathology: A meta-analytic review. Clinical Psychology Review, 30(2), 217-237. https://doi.org/ 10.1016/j.cpr.2009.11.004

American Psychiatric Association. (1994). Diagnostic and statistical manual of mental disorders (4th ed.). Washington, DC: Author.

Ayduk, O., \& Kross, E. (2008). Enhancing the pace of recovery: Selfdistanced analysis of negative experiences reduces blood pressure reactivity. Psychological Science, 19(3), 229-231. https://doi.org/ 10.1111/j.1467-9280.2008.02073.x

Banks, S. J., Eddy, K. T., Angstadt, M., Nathan, P. J., \& Luan Phan, K. (2007). Amygdala-frontal connectivity during emotion regulation. Social Cognitive and Affective Neuroscience, 2(4), 303-312.

Brosschot, J. F., \& Thayer, J. F. (2003). Heart rate response is longer after negative emotions than after positive emotions. International Journal of Psychophysiology, 50(3), 181-187. https://doi.org/10. 1016/S0167-8760(03)00146-6

Buhle, J. T., Silvers, J. A., Wager, T. D., Lopez, R., Onyemekwu, C., Kober, H., ... Ochsner, K. N. (2013). Cognitive reappraisal of emotion: A meta-analysis of human neuroimaging studies. Cerebral Cortex. https://doi.org/10.1093/cercor/bht154

Christoff, K., \& Gabrieli, J. D. E. (2000). The frontopolar cortex and human cognition: Evidence for a rostrocaudal hierarchical organization within the human prefrontal cortex. Psychobiology, 28(2), 168-186.

Cohen, M. S. (1997). Parametric analysis of fMRI data using linear systems methods. NeuroImage, 6(2), 93-103.

Corbetta, M., Patel, G., \& Shulman, G. L. (2008). The reorienting system of the human brain: From environment to theory of mind. Neuron, 58(3), 306-324. https://doi.org/10.1016/j.neuron.2008.04.017

Corbetta, M., \& Shulman, G. L. (2002). Control of goal-directed and stimulus-driven attention in the brain. Nature Reviews Neuroscience, 3(3), 201-215.

Cox, R. W. (1996). AFNI: Software for analysis and visualization of functional magnetic resonance neuroimages. Computers and Biomedical Research, 29, 162-173.

Dan-Glauser, E. S., \& Gross, J. J. (2015). The temporal dynamics of emotional acceptance: Experience, expression, and physiology. Biological Psychology, 108, 1-12. https://doi.org/10.1016/j. biopsycho.2015.03.005

Di Nardo, P. A., Brown, T. A., \& Barlow, D. H. (1994). Anxiety Disorders Interview Schedule for DSM-IV: Lifetime version (ADIS-IV-L). New York, NY: Oxford University Press.

Ellard, K. K., Barlow, D. H., Whitfield-Gabrieli, S., Gabrieli, J. D. E., \& Deckersbach, T. (2017). Neural correlates of emotion acceptance vs. 
worry or suppression in generalized anxiety disorder. Social Cognitive and Affective Neuroscience, 12(6), 1009-1021. https:// doi.org/10.1093/scan/nsx025

Fan, J., McCandliss, B. D., Fossella, J., Flombaum, J. I., \& Posner, M. I. (2005). The activation of attentional networks. NeuroImage, 26(2), 471-479.

Forman, S. D., Cohen, J. D., Fitzgerald, M., Eddy, W. F., Mintun, M. A., \& Noll, D. C. (1995). Improved assessment of significant activation in functional magnetic resonance imaging (fMRI): Use of a clustersize threshold. Magnetic Resonance in Medicine, 33, 636-647.

Garnefski, N., Kraaij, V., \& Spinhoven, P. (2002). Manual for the use of the cognitive emotion regulation questionnaire: English version. Leiderdorp, The Netherlands: DATEC.

Gerritsen, R. J. S., \& Band, G. P. H. (2018). Breath of life: The respiratory vagal stimulation model of contemplative activity. Frontiers Human Neuroscience, 12, 397. https://doi.org/10.3389/fnhum.2018.00397

Giuliani, N. R., McRae, K., \& Gross, J. J. (2008). The up- and downregulation of amusement: Experiential, behavioral, and autonomic consequences. Emotion, 8(5), 714-719. https://doi.org/10.1037/ a0013236

Glover, G. H., \& Law, C. S. (2001). Spiral-in/out BOLD fMRI for increased SNR and reduced susceptibility artifacts. Magnetic Resonance in Medicine, 46(3), 515-522.

Goldin, P., Ziv, M., Jazaieri, H., Hahn, K., \& Gross, J. J. (2013). MBSR vs. aerobic exercise in social anxiety: fMRI of emotion regulation of negative self-beliefs. Social Cognitive Affective Neuroscience, 8(1), 65-72. https://doi.org/10.1093/scan/nss054

Goldin, P. R., Manber-Ball, T., Werner, K., Heimberg, R., \& Gross, J. J. (2009). Neural mechanisms of cognitive reappraisal of negative selfbeliefs in social anxiety disorder. Biological Psychiatry, 66(12), 1091-1099. https://doi.org/10.1016/j.biopsych.2009.07.014

Goldin, P. R., McRae, K., Ramel, W., \& Gross, J. J. (2008). The neural bases of emotion regulation: Reappraisal and suppression of negative emotion. Biological Psychiatry, 63(6), 577-586.

Goosheh, B., Ravanbakhsh, M., Salavati, M., Ebrahimi Takamjani, I., Akhbari, B., \& Kahlaee, A. H. (2017). Attention-demand effects on respiration in chronic low back pain patients. Journal of Bodywork and Movement Therapies, 21(4), 788-793. https://doi. org/10.1016/j.jbmt.2016.11.016

Gross, J. J. (2002). Emotion regulation: Affective, cognitive, and social consequences. Psychophysiology, 39(3), 281-291. https://doi.org/ $10.1017 /$ S0048577201393198

Gross, J. J. (2013). Emotion regulation: Taking stock and moving forward. Emotion, 13(3), 359-365. https://doi.org/10.1037/a0032135

Gross, J. J. (2015). Emotion Regulation: Current status and future prospects. Psychological Inquiry, 26(1), 1-26. https://doi.org/10.1080/ 1047840X.2014.940781

Hayes, S. C., Strosahl, K. D., \& Wilson, K. G. (1999). Acceptance and commitment therapy: An experiential approach to behavior change. New York, NY: Guilford Press.

Hofmann, S. G., Heering, S., Sawyer, A. T., \& Asnaani, A. (2009). How to handle anxiety: The effects of reappraisal, acceptance, and suppression strategies on anxious arousal. Behaviour Research and Therapy, 47(5), 389.

Holzman, J. B., \& Bridgett, D. J. (2017). Heart rate variability indices as bio-markers of top-down self-regulatory mechanisms: A metaanalytic review. Neuroscience and Biobehavioral Review, 74(Pt. A), 233-255. https://doi.org/10.1016/j.neubiorev.2016.12.032

Kross, E., \& Ayduk, O. (2008). Facilitating adaptive emotional analysis: Distinguishing distanced-analysis of depressive experiences from immersed-analysis and distraction. Personality and Social Psychology Bulletin, 34(7), 924-938. https://doi.org/10.1177/ 0146167208315938

Lebois, L. A. M., Papies, E. K., Gopinath, K., Cabanban, R., Quigley, K. S., Krishnamurthy, V., . . . Barsalou, L. W. (2015). A shift in perspective: Decentering through mindful attention to imagined stressful events. Neuropsychologia, 75, 505-524. https://doi.org/ 10.1016/j.neuropsychologia.2015.05.030

LeDoux, J. (2007). The amygdala. Current Biology, 17(20), R868-874.

Levy, B. J., \& Wagner, A. D. (2011). Cognitive control and right ventrolateral prefrontal cortex: Reflexive reorienting, motor inhibition, and action updating. Annals of the New York Academy of Sciences, 1224(1), 40-62. https://doi.org/10.1111/j.1749-6632.2011.05958.x

Liverant, G. I., Brown, T. A., Barlow, D. H., \& Roemer, L. (2008). Emotion regulation in unipolar depression: The effects of acceptance and suppression of subjective emotional experience on the intensity and duration of sadness and negative affect. Behaviour Research and Therapy, 46(11), 1201-1209. https://doi.org/10. 1016/j.brat.2008.08.001

Morawetz, C., Bode, S., Baudewig, J., Kirilina, E., \& Heekeren, H. R. (2016). Changes in effective connectivity between dorsal and ventral prefrontal regions moderate emotion regulation. Cerebral Cortex, 26(5), 1923-1937. https://doi.org/10.1093/cercor/bhv005

Ochsner, K. N., Silvers, J. A., \& Buhle, J. T. (2012). Functional imaging studies of emotion regulation: A synthetic review and evolving model of the cognitive control of emotion. Annals of the New York Academy of Sciences, 1251, E1-E24.

Oldfield, R. C. (1971). The assessment and analysis of handedness: The Edinburgh Inventory. Neuropsychologia, 9(1), 97-113. https://doi. org/10.1016/0028-3932(71)90067-4

Olson, I. R., McCoy, D., Klobusicky, E., \& Ross, L. A. (2013). Social cognition and the anterior temporal lobes: A review and theoretical framework. Social Cognitive and Affective Neuroscience, 8(2), 123 133. https://doi.org/10.1093/scan/nss 119

Pascoe, M. C., Thompson, D. R., Jenkins, Z. M., \& Ski, C. F. (2017). Mindfulness mediates the physiological markers of stress: Systematic review and meta-analysis. Joural of Psychiatry Research, 95, 156-178. https://doi.org/10.1016/j.jpsychires.2017. 08.004

Smith, S. M., \& Nichols, T. E. (2009). Threshold-free cluster enhancement: Addressing problems of smoothing, threshold dependence and localisation in cluster inference. NeuroImage, 44(1), 83-98. https://doi.org/10.1016/j.neuroimage.2008.03.061

Smoski, M. J., Keng, S. L., Ji, J. L., Moore, T., Minkel, J., \& Dichter, G. S. (2015). Neural indicators of emotion regulation via acceptance vs. reappraisal in remitted major depressive disorder. Social Cognitive and Affective Neuroscience, 10(9), 1187-1194. https://doi.org/10. 1093/scan/nsv003

Stange, J. P., Hamilton, J. L., Fresco, D. M., \& Alloy, L. B. (2017). Flexible parasympathetic responses to sadness facilitate spontaneous affect regulation. Psychophysiology, 54(7), 1054-1069. https:// doi.org/10.1111/psyp.12856

Talairach, J., \& Tournoux, P. (1988). Co-planar stereotaxic atlas of the human brain. New York, NY: Thieme.

Touroutoglou, A., Andreano, J. M., Barrett, L. F., \& Dickerson, B. C. (2015). Brain network connectivity-behavioral relationships exhibit trait-like properties: Evidence from hippocampal connectivity and memory. Hippocampus, 25(12), 1591-1598. https://doi.org/10. 1002/hipo. 22480

Wolgast, M., Lundh, L. G., \& Viborg, G. (2011). Cognitive reappraisal and acceptance: An experimental comparison of two emotion regulation strategies. Behaviour Research and Therapy, 49(12), 858866. https://doi.org/10.1016/j.brat.2011.09.011

Wolgast, M., Lundh, L. G., \& Viborg, G. (2013). Cognitive restructuring and acceptance: An empirically grounded conceptual analysis. Cognitive Therapy and Research, 37(2), 340-351. https://doi.org/ 10.1007/s10608-012-9477-0

Zvolensky, M. J., \& Eifert, G. H. (2001). A review of psychological factors/processes affecting anxious responding during voluntary hyperventilation and inhalations of carbon dioxide-enriched air. Clinical Psychology Review, 21(3), 375-400. 